\title{
A splitting theorem for multipeak path algebras
}

by

Daniel Simson (Toruń)

Abstract. Let $F$ be a division ring, 2 a locally finite quiver, $\Omega$ an admissible ideal [4] in the path algebra $F 2$ of $i 2$ and suppose that the bound quiver algebra $R=F(2, \Omega):=F 2 / \Omega$ of $(2, \Omega)$ [5] is a right multipeak algebra in the sense that $\operatorname{soc}\left(R_{R}\right)$ is an essential and projective submodule of $R_{R}$ [22]. Let $\bmod _{\mathrm{sp}}(R)$ be the category of finitely generated socle projective right $R$-modules. Following an idea in $[15,11]$ we prove that if the bound quiver $(2, \Omega)$ admits a splitting decomposition (3.2) explained in Fig. 6 then there are two proper bound subquivers $\left(\mathscr{f}^{\prime}, \Omega^{\prime}\right)$ $\left(\mathscr{J}^{\prime \prime}, \Omega^{\prime \prime}\right)$ of $(2, \Omega)$ and full faithful cxact embeddings

$$
\bmod _{\mathrm{sp}} F\left(\mathscr{J}^{\prime}, \Omega^{\prime}\right) \stackrel{\boldsymbol{T}}{\rightarrow} \bmod _{\mathrm{sp}}(R) \stackrel{\underline{L}}{-} \bmod _{\mathrm{sp}} F\left(\mathscr{J}^{\prime \prime}, \Omega^{\prime \prime}\right)
$$

such that any indecomposable module in $\bmod _{\text {sp }}(R)$ belongs either to $\operatorname{Im} T$ or to $\operatorname{Im} L$. The functors $T$ and $L$ carry Auslander-Reiten sequences to Auslander-Reiten sequences and induce a splitting of the Auslander-Reiten translation quiver $\Gamma_{\mathrm{sp}}(R)$ of $\bmod _{\mathrm{sp}}(R)$ (see 3.10, 3.11 and Fig. 7).

1. Introduction. Multipeak rings and socle projective modules play an important role in the study of matrix problems and vector space categories [21-24] as well as in the classification of indecomposable modules over finite-dimensional algebras [17-19] and lattices over orders [20]. One can prove that if $R=F(2, \Omega)$ is a simply connected [0] right peak algebra [21] and for any vertex $i \in \mathcal{Q}_{0}$ having no oriented path $w: i \rightarrow i$ in $(2, \Omega)$ there is a unique path (up to a scalar) from $i$ to the unique $\operatorname{sink}$ in $(2, \Omega)$ then $\bmod _{\text {sp }}(R)$ is equivalent to the category $I$-sp of $I$-spaces, where $I$ is a poset, and therefore its representation type can be determined by the criteria of Kleiner [10] and Nazarova [14]. In case $R$ is not simply connected there exists a universal Galois covering $(\widetilde{Q}, \tilde{\Omega})$ of $(2, \Omega)[5,8,13]$ such that $\tilde{R}=F(\tilde{\mathscr{Q}}, \tilde{\Omega})$ is a multipeak algebra and by $[22$; Theorem $1.10]$ the push-down functor [4] reduces the study of $\operatorname{ind}_{\mathrm{sp}}(R)$ to the study of $\operatorname{ind}_{\mathrm{sp}}(\tilde{R})$, where $\operatorname{ind}_{\mathrm{sp}}(R)$ is the full subcategory of $\bmod _{\mathrm{sp}}(R)$ consisting of pairwise nonisomorphic representatives of indecomposable modules (see 4.2 and 4.3). In order to apply the technique developed in $[4,7,8]$ one needs a description of supports of modules in $\operatorname{ind}_{\mathrm{sp}}(\widetilde{R})$. This is one of the motivations for studying splitting decompositions of $(\tilde{Q}, \tilde{\Omega})$ because in many situations our splitting theorems 3.10 and 3.11 allow us to determine

Supported by Polish scientific grant RP.I.10. 
the supports of modules in ind $\mathrm{sp}_{\mathrm{sp}}(\tilde{R})$ as well as the Auslander-Reiten quiver $\boldsymbol{\Gamma}_{\mathrm{sp}}(\tilde{R})$. This technique is applied in $[24,26,27]$ where among other things we determine the sp-representation type of a class of right peak algebras $R$, which are incidence algebras of bipartite posets (comp. [16]). We also determine $\Gamma_{\mathrm{sp}}(R)$ for any such algebra $R$ which is sp-representation-finite. One of our tools applied in [26] is the splitting theorem. Another application of our splitting theorem is given in Section 4, where we determine the sp-representation type of $R=F(2, \Omega)$ in case $(2, \Omega)$ is a multiserial tree. The splitting theorem reduces the problem to a corresponding problem for $I$-spaces (comp. [5]).

In Section 2 we collect basic definitions and notation. In particular a reflection duality $D^{\bullet}: \bmod _{\mathrm{sp}}(R) \rightarrow \bmod _{\mathrm{sp}}\left(R^{\bullet}\right)^{\circ p}$ is defined. Moreover, given $(2, \Omega)$ we define a bound quiver $\left(\mathscr{Q}^{\bullet}, \Omega^{\bullet}\right)$ such that $(F(\mathscr{Q}, \Omega))^{\bullet} \cong F\left(\mathscr{Q}^{\bullet}, \Omega^{\bullet}\right)$ provided $\operatorname{dim}_{F} e_{i} F(\mathscr{Q}, \Omega) e_{p} \leqslant 1$ for all $i \in \mathscr{Q}_{0}$ and all sinks $p \in \mathscr{Q}_{0}$ (Corollary 2.22).

In Section 3 we present our main results (Theorem 3.10 and Corollary 3.11). As a consequence we get in Corollary 3.13 a generalization of an edge reduction given by Ringel and Roggenkamp [19]. Section 4 contains various examples illustrating our main results and their applications.

Throughout this paper $\bmod (R)$ denotes the category of finitely generated right $R$-modules. The projective cover and the injective envelope of a module $X$ in $\bmod (R)$ will be denoted by $P(X)$ and $E_{R}(X)$, respectively. We call $R$ sp-representation-finite (resp. -tame) if $\operatorname{ind}_{\mathrm{sp}}(R)$ is finite (resp. $\bmod _{\mathrm{sp}}(R)$ is tame in the sense of [6]).

I would like to thank Dr. Th. Weichert for some corrections of the preliminary version of this paper.

2. Preliminaries. We collect here the results and notation we need for our splitting theorem. Throughout we suppose that

$$
R=\underset{i \in I_{R}}{\oplus} e_{i} R
$$

is a basic semiperfect $F$-algebra (in general without identity) and $\left\{e_{i}\right\}_{i \in I_{R}}$ is a fixed set of primitive orthogonal idempotents of $R$. We suppose that $R$ is locally bounded (i.e. $\sum_{j} \operatorname{dim}_{F} e_{i} R e_{j}$ and $\sum_{j} \operatorname{dim}_{F} e_{j} R e_{i}$ are finite for every $\left.i \in I_{R}[4]\right)$ and $R$ is a right multipeak algebra [22] with the set $\left\{e_{p}\right\}_{p \in p\left(I_{R}\right)}, \boldsymbol{p}\left(I_{R}\right) \subseteq I_{R}$, of peak idempotents (i.e. $e_{p} R$ is simple for any $p \in p\left(I_{R}\right), \operatorname{soc}\left(e_{j} R\right)$ is essential in $e_{j} R$ and isomorphic to a finite direct sum of modules $e_{p} R, p \in p\left(I_{R}\right)$, for all $\left.j \in I_{R}\right)$. It follows that

$$
R=\left(\begin{array}{cc}
A & { }_{A} N_{B} \\
0 & B
\end{array}\right)
$$

where $A=e R e, B=e_{*} R e_{*}, N=e R e_{*}$ and

$$
e_{*}=\sum_{p \in p\left(I_{R}\right)} e_{p}, \quad e=\sum_{j \in I_{R}-p\left(I_{R}\right)} e_{j} .
$$

Here the sums are formal if the index set is infinite. By $\left(\sum_{j \in J} e_{j}\right) R$ we shall mean $\sum_{j \in J} e_{j} R$. Note that $B$ is a direct sum of division rings $B_{p}=e_{p} R e_{p},{ }_{A} N$ is $A$-faithful and $\operatorname{dim}_{F} e_{j} N$ is finite for $j \in I_{R}$.
In the case $\left|\boldsymbol{p}\left(I_{R}\right)\right|=1, e_{*}$ is primitive and we call $R$ a right peak algebra with a peak $e_{*} R[21]$.

Following $[21 ; 2.6],[3 ;$ p. 906], [9] we associate with $R$ two reflection forms

$$
R^{\nabla}=\left(\begin{array}{cc}
B & D\left(R e_{*}\right) \\
0 & R
\end{array}\right) \cong\left(\begin{array}{ccc}
B & \tilde{N} & B \\
0 & A & N \\
0 & 0 & B
\end{array}\right), \quad R^{\nabla}=\left(\begin{array}{cc}
B & \tilde{N} \\
0 & A
\end{array}\right) \cong R^{\mathbf{\nabla}} / R^{\mathbf{\nabla}} e_{*} R^{\mathbf{\nabla}},
$$

where $D(-)=\operatorname{Hom}_{F}(-, F),{ }_{B} \tilde{N}_{A}=\operatorname{Hom}_{B}\left({ }_{A} N_{B}, B\right) \cong D\left({ }_{A} N_{B}\right)$ and multiplication in $R^{\mathbf{v}}$ is given by the evaluation map ${ }_{B} \tilde{N} \otimes_{A} N_{B} \rightarrow B$. It is clear that $R^{\mathbf{v}}$ is a left multipeak algebra with left peak idempotents

$$
e_{p}^{-}=\left(\begin{array}{ll}
e_{p} & 0 \\
0 & 0
\end{array}\right)
$$

where $p \in p\left(I_{R}\right)$ and $e_{p}$ is considered here as an element of $B$. We put

$$
e_{*}^{-}=\sum_{p \in p\left(I_{R}\right)} e_{p}^{-} .
$$

Following $[21,23]$ we define a pair of reflection functors

$$
\bmod _{\mathrm{sp}}(R) \underset{\nabla_{+}}{\stackrel{\nabla_{-}}{\longrightarrow}} \bmod _{\mathrm{ti}}(R \nabla)
$$

where $\bmod _{\mathrm{ti}}\left(R^{\nabla}\right)$ is the category of finitely generated top injective right $R$-modules. Given $X_{R}=\left(X_{A}^{\prime}, X_{B}^{\prime \prime}, \varphi: X^{\prime} \otimes_{A} N_{B} \rightarrow X_{B}^{\prime \prime}\right), Y_{R}=\left(Y_{B}^{\prime}, Y_{A}^{\prime \prime}, \psi: Y^{\prime} \otimes_{B} N_{A} \rightarrow Y_{A}^{\prime \prime}\right)$ we put

$$
\nabla_{-}\left(X_{R}\right)=\left(X_{B}^{\prime \prime}, \operatorname{Coker} \varphi^{\prime}, \tilde{\varphi}\right), \quad \nabla_{+}(Y)=\left(\operatorname{Ker} \psi^{\prime}, Y_{B}^{\prime}, \tilde{\psi}\right),
$$

where $\varphi^{\prime}$ and $\psi^{\prime}$ are the composed maps $X_{A}^{\prime}{ }_{\rightarrow} \rightarrow \operatorname{Hom}_{B}\left(N_{B}, X_{B}^{\prime \prime}\right) \cong X^{\prime \prime} \otimes_{B} \tilde{N}_{A}$ and $\operatorname{Hom}_{B}\left({ }_{A} N_{B}, Y_{B}^{\prime}\right) \cong Y^{\prime} \otimes_{B} \tilde{N}_{A}{ }^{\psi} \rightarrow Y_{A}^{\prime \prime}$ respectively, $\bar{\varphi}$ is adjoint to $\varphi, \tilde{\varphi}$ is the cokernel map and $\tilde{\psi}$ is adjoint to the natural embedding $\operatorname{Ker} \psi^{\prime} \hookrightarrow \operatorname{Hom}_{B}\left({ }_{A} N_{B}, Y_{B}^{\prime}\right)$. The functors are defined on maps in a natural way.

Proposition 2.5. (a) $R^{\nabla}$ is a left multipeak algebra with left peak idempotents $e_{p}^{-}$, $p \in \boldsymbol{p}\left(I_{R}\right)$, and a right multipeak algebra with right peak idempotents $e_{p}, p \in \boldsymbol{p}\left(I_{R}\right)$. Moreover, $e_{p}^{-} R^{\boldsymbol{\nabla}} \cong E\left(e_{p} R^{\boldsymbol{\nabla}}\right)$ for $p \in p\left(I_{R}\right)$ and $E\left(R^{\mathbf{\nabla}}\right)$ is projective. If $X$ is in ind $_{\mathrm{sp}}\left(R^{\mathbf{\nabla}}\right), Y$ is in $\operatorname{ind}_{\mathrm{ti}}\left(R^{\nabla}\right)$ and $X e_{*}^{-} \neq 0, Y e_{*} \neq 0$ then $X \cong E\left(e_{p} R^{\nabla}\right), Y \cong E\left(e_{q} R^{\nabla}\right)$ for some $p, q \in p\left(I_{R}\right)$.

(b) $\nabla_{\ldots}$ and $\nabla_{+}$are equivalences of categories preserving exactness and inverse to each other. $X_{R}$ is sp-injective if and only if $\nabla_{-}\left(X_{R}\right)$ is injective in $\bmod \left(R^{\nabla}\right)$ (see below for definition)

(c) Given $X_{R}$ in $\bmod _{\mathrm{sp}}(R)$ and $Y$ in $\bmod _{\mathrm{ii}}\left(R^{\nabla}\right)$ we have

$$
\nabla_{-}\left(X_{R}\right) \cong \operatorname{Coker}\left(X \hookrightarrow E_{R} \mathbf{\nabla}(X)\right), \quad \nabla_{+}(Y) \cong \operatorname{Ker}\left(P_{R} \mathbf{\nabla}(Y) \rightarrow Y\right)
$$

where $X$ and $Y$ are considered as $R^{\mathbf{\nabla}}$-modules via the epimorphisms $R \leftarrow R^{\nabla} \rightarrow R^{\nabla}$.

Proof. (a) and (b) can be proved by applying arguments in the proof of Propositions 2.6 and 2.8 in [21] and in [3; Proposition 1.6].

(c) By (a) indecomposable summands of $E_{R} \mathbf{\nabla}\left(X_{R}\right)$ are summands of $e_{*}^{-} R^{\mathbf{\nabla}}$ which is 
the row ideal $(B, \tilde{N}, B)$ in the form (2.3). It follows that up to isomorphism the embedding $X \leftrightharpoons E_{R} \nabla(X)$ is given by the natural monomorphism $\left(0, \varphi^{\prime}, 0\right)$ : $\left(0, X_{A}^{\prime}, X_{B}^{\prime \prime}\right)$ $\rightarrow\left(X_{B}^{\prime \prime}, X^{\prime} \otimes_{A} \tilde{N}_{B}, X_{B}^{\prime \prime}\right)$, where $\varphi^{\prime}$ is the map in the formula defining $\nabla_{-}\left(X_{R}\right)$. Hence the first isomorphism in (c) follows. The second one can be established in a similar way.

We recall that $X$ in $\bmod _{\mathrm{sp}}(R)$ is sp-injective if $X$ is injective with respect to monomorphisms $f: Z \rightarrow Z^{\prime}$ in $\bmod _{\mathrm{sp}}(R)$ such that Coker $f$ is in $\bmod _{\mathrm{sp}}(R)$ [21]. An indecomposable sp-injective module $X$ in $\bmod _{\mathrm{sp}}(R)$ is said to be hereditary if every module $X^{\prime}$ in $\operatorname{ind}_{\mathrm{sp}}(R)$ such that $\operatorname{Hom}_{R}\left(X, X^{\prime}\right) \neq 0$ is sp-injective.

We shall call $R^{\bullet}=\left(R^{\nabla}\right)^{\text {op }}$ the reflection dual algebra to $R$ and the functor

$$
D_{R}^{\bullet}:=D P_{-}: \bmod _{\mathrm{sp}}(R) \rightarrow \bmod _{\mathrm{sp}}\left(R^{\bullet}\right)
$$

will be called the reflection duality. It follows from Proposition 2.5 that $D_{R}^{\bullet}$ is a duality, there is an algebra isomorphism $R^{\bullet \bullet} \cong R, D_{R}^{\bullet \bullet} \cong \nabla_{+} D \cong\left(D_{R}^{\bullet}\right)^{-1}$ and $X_{R}$ is hereditary sp-injective if and only if $D_{R}^{\bullet}\left(X_{R}\right)$ is hereditary projective [28], i.e. every submodule of $D_{R}^{\bullet}\left(X_{R}\right)$ is projective. The modules

$$
Q^{(p)}=D_{R^{\bullet}}^{\bullet}\left(e_{p}^{-} R^{\bullet}\right), \quad p \in p\left(I_{R}\right), \quad Q^{(j)}=D_{R}^{\bullet \bullet}\left(e_{j} R^{\bullet}\right), \quad j \in I_{R}-p\left(I_{R}\right)
$$

form a complete set of nonisomorphic indecomposable sp-injective modules in $\bmod _{\mathrm{sp}}(R)$.

It is known that there are Auslander--Reiten sequences in $\bmod (R)$ [4]. Then by $[2$, $21,23]$ we get

Proposition 2.8. If $R$ is an $F$-algebra as above then for every nonprojective module $X$ in $\operatorname{ind}_{\mathrm{sp}}(R)$ and for every non-sp-injective module $Y$ in $\operatorname{ind}_{\mathrm{sp}}(R)$ there are Auslander-Reiten sequences in $\bmod _{\mathrm{sp}}(R)$

$$
0 \rightarrow X \rightarrow Z \rightarrow \Delta^{-}(X) \rightarrow 0, \quad 0 \rightarrow \Delta(Y) \rightarrow U \rightarrow Y \rightarrow 0
$$

which are unique up to isomorphism and $\Delta^{-}(X), \Delta(Y)$ are indecomposable.

Now suppose $J \subseteq I_{R}$ is such that $p(J) \subseteq p\left(I_{R}\right)$ and $R_{J}=v R v$ with $v=\sum_{j \in J} e_{j}$ is a right multipeak algebra with peak idempotents $e_{q}, q \in p(J)$. Following [1], [22; 1.14], [23] we consider three functors

$$
\bmod _{\mathrm{sp}}(v R v) \stackrel{\boldsymbol{T}_{v}, \boldsymbol{L}_{v}}{\stackrel{\boldsymbol{r}_{v}}{\rightleftarrows}} \bmod _{\mathrm{sp}}(R)
$$

where $r_{v}(X)=X v, T_{v}(Y)=Y \otimes_{v R v} v R$ and $L_{v}(Y)=\operatorname{Hom}_{v R v}(R v, Y)$.

In view of [1] and [22; Corollary 1.16] we get

Proposition 2.11. If $J \subseteq I_{R}$ and $v$ are as above then

(a) $\boldsymbol{T}_{v}$ and $\boldsymbol{L}_{v}$ are fully faithful embeddings, $\boldsymbol{r}_{v}$ is exact, $\boldsymbol{T}_{v}$ is left adjoint to $\boldsymbol{r}_{v}, \boldsymbol{L}_{v}$ is right adjoint to $\boldsymbol{r}_{v}$ and $\boldsymbol{r}_{v} \boldsymbol{T}_{v} \cong \mathrm{id} \cong \boldsymbol{r}_{v} \boldsymbol{L}_{v}$

(b) $T_{v}\left(e_{j} R v\right) \cong e_{j} R$ for $j \in J, T_{v}$ preserves projective covers and $\operatorname{Im} T_{v}$ is the full subcategory of $\bmod _{\mathrm{sp}}(R)$ consisting of modules $X$ such that $P(X) \cong \oplus_{j \in J}\left(e_{j} R\right)^{s_{J}}$.
To any $X$ in $\operatorname{ind}_{\mathrm{sp}}(R)$ we associate two integral vectors [21; Section 3] (2.12)

$$
\operatorname{dim}(X)=\left(x_{i}\right)_{i \in I_{R}}, \quad \operatorname{cdn}(X)=\left(s_{i}\right)_{i \in I_{R}},
$$

where $x_{i}=\operatorname{dim}_{F} X e_{i}, s_{p}=\operatorname{dim}_{F} X e_{p}$ for $p \in p\left(I_{R}\right)$ and given $j \in I_{R}-p\left(I_{R}\right), s_{j}$ is such that

$$
P(X) \cong \bigoplus\left(e_{i} R\right)^{s_{i}} .
$$

We call $\operatorname{dim}(X)$ and $\operatorname{cdn}(X)$ the dimension vector of $X$ and the coordinate vector of $X$ respectively. The sets

$$
\operatorname{supp}(X)=\left\{i \in I_{R} ; x_{i} \neq 0\right\}, \quad \operatorname{csup}(X)=\left\{i \in I_{R} ; s_{i} \neq 0\right\}
$$

are called the support and the coordinate support of $X$, respectively. Note that $\operatorname{csup}(X)$ is finite. $X$ in $\operatorname{ind}_{\mathrm{sp}}(R)$ is called sp-sincere $[11]$ if $\operatorname{csup}(X)=I_{R} . R$ is said to be sp-sincere if there is an sp-sincere module in $\operatorname{ind}_{\mathrm{sp}}(R)$.

It is clear that if $X$ is in $\operatorname{ind}_{\mathrm{sp}}(R)$ and $v=\sum_{j \in \operatorname{csup}(X)} e_{j}$ then $v R v$ is a right multipeak algebra, $r_{v}(X)$ is an sp-sincere $v R v$-module and $T_{v} r_{v}(X) \cong X$. It follows that up to the


module over $v R v \subseteq R$.

Note also that if $J \subseteq I_{R}$ and $v$ are as in Proposition 2.11 then we have

$$
\left.\operatorname{cdn}\left(\boldsymbol{T}_{v}(Y)\right)\right|_{J}=\operatorname{cdn}(Y), \quad Y \in \operatorname{ind}_{\mathrm{sp}}(v R v) .
$$

In describing a bound quiver of $R^{\mathbf{V}}$ in terms of the algebra $R$ the following simple lemma will be useful:

LEMMA 2.15. Let

$$
R=\left(\begin{array}{ll}
A & N \\
0 & B
\end{array}\right)
$$

be a locally bounded right multipeak $F$-algebra (2.1) and let $R^{\nabla}, R^{\nabla}$ be the reflection forms (2.3) of $R$. In the notation above we have

$$
\begin{aligned}
e_{i} R^{\nabla} e_{j}=e_{i} R^{\nabla} e_{j} & =e_{i} R e_{j} & & \text { for } i \in I_{R}-p\left(I_{R}\right), j \in I_{R}, \\
e_{p}^{-} R^{\nabla} e_{q} & =0 & & \text { for } p \neq q, p, q \in \boldsymbol{p}\left(I_{R}\right), \\
& =e_{p} R e_{p} & & \text { for } p=q .
\end{aligned}
$$

(b) There is a bimodule isomorphism $\sigma:{ }_{B} \tilde{N}_{A} \rightarrow D\left({ }_{A} N_{B}\right)$ which induces F-linear isomorphisms

$$
\sigma_{p j}: e_{p}^{-} \tilde{N} e_{j}=e_{p}^{-} R^{\nabla} e_{j}=e_{p}^{-} R^{\nabla} e_{j} \simeq D\left(e_{j} N e_{p}\right)=D\left(e_{j} R e_{p}\right)
$$

for all $p \in p\left(I_{R}\right), j \in I_{R}-p\left(I_{R}\right)$, such that given $i \in I_{R}-p\left(I_{R}\right)$ the diagram

$$
\begin{array}{rrr}
e_{p}^{-} \tilde{N} e_{j} \otimes e_{j} R e_{i} \stackrel{\sigma_{p j} \otimes 1}{\longrightarrow} D\left(e_{j} N e_{p}\right) \otimes e_{j} R e_{i} \\
\downarrow \mu_{p j i} & & \downarrow \tilde{c}_{p j i} \\
e_{p}^{-} \tilde{N} e_{i} & \stackrel{\sigma_{p l} \otimes 1}{\longrightarrow} & D\left(e_{i} N e_{p}\right)
\end{array}
$$


is commutative, where $D(-)=\operatorname{Hom}_{F}(-, F), \mu_{p j i}(g \otimes y)=g y, \tilde{c}_{p j i}(f \otimes y)(n)=f(y n)$ for $n \in e_{i} N e_{p}, y \in e_{j} R e_{i}, f \in D\left(e_{j} N e_{p}\right), g \in e_{p}^{-} \tilde{N} e_{j}$.

The proof is easy and is left to the reader.

Now suppose that $R=F(\mathscr{Q}, \Omega)$ is a bound quiver algebra. In this case we take $I_{R}=\mathscr{Q}_{0}$ (the set of vertices of $\mathscr{Q}$ ) and we take for $\left\{e_{i}\right\}_{i \in \mathscr{Q}_{0}}$ the standard set of idempotents which are the trivial paths $\mathbf{1}_{i}: i \rightarrow i$ in $\mathscr{Q}$. Then $p\left(I_{R}\right)$ is the set $p(\mathscr{Q})$ of all sinks in $\mathscr{Q}$ and $R$ is a right multipeak algebra if and only if for any $k_{1}, \ldots, k_{t} \in F$ and paths $w_{1}, \ldots, w_{t}$ : $i \rightarrow j$ such that $w=k_{1} w_{1}+\ldots+k_{t} w_{t} \notin \Omega$ there exist $p \in p(\mathscr{2})$ and a path $u: j \rightarrow p$ such that $w u \notin \Omega$. If $(2, \Omega)$ has the above property we call it a right multipeak bound quiver.

Definition 2.16. Suppose that $(\mathscr{Q}, \Omega)$ is a right multipeak bound quiver, $\Omega$ is an admissible ideal in $F \mathscr{Q}, R=F(\mathscr{Q}, \Omega)$ and suppose that $R$ is peak $\tilde{A}_{1}$-free, i.e. $\operatorname{dim}_{F} e_{i} R e_{p} \leqslant 1$ for all $i \in \mathscr{Q}_{0}$ and $p \in p(2)$ (in the notation above). We define two reflection forms $\left(\mathscr{Q}^{\nabla}, \Omega^{\nabla}\right)$ and $\left(\mathscr{Q}^{\nabla}, \Omega^{\nabla}\right)$ of $(\mathscr{Q}, \Omega)$ as follows. Let

$$
\mathscr{Q}_{0}^{\mathbf{V}}=p(\mathscr{2})^{-} \dot{\sim} \mathscr{Q}_{0}, \quad \mathscr{Q}_{0}^{\nabla}=\mathscr{Q}_{0}^{\nabla}-p(\mathscr{2})
$$

where $\boldsymbol{p}(2)^{-}$consists of vertices $p^{-}$with $p \in \boldsymbol{p}(\mathscr{2})$ and $\dot{\cup}$ means disjoint union. The sets of edges in $\mathscr{Q}^{\nabla}$ and in $\mathscr{Q}^{\nabla}$ between the vertices in $\mathscr{Q}_{0}$ remain the same as in $\mathscr{2}$. For any $i \in \mathscr{Q}_{0}-\boldsymbol{p}(\mathscr{2})$ and $p \in \boldsymbol{p}(\mathscr{2})$ such that $e_{i} R e_{p} \neq 0$ we fix a path $u_{i p}: i \rightarrow p$ in $\mathscr{Q}$ which does not belong to $\Omega$. We define a unique edge

$$
u_{i p}^{-}: p^{-} \rightarrow i
$$

in $\mathscr{Q}^{\nabla}$ (and in $\mathscr{Q}^{\nabla}$ ) if $u_{i p}$ is maximal modulo $\Omega$ in the sense that $u u_{i p} \in \Omega$ for every edge $u$ in 2 ending at $i$ (see Fig. 0 ).

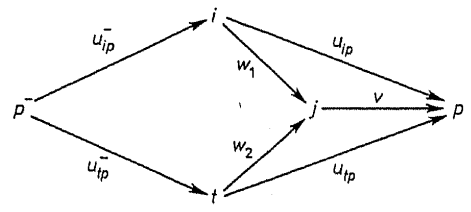

Fig. 0

We define $\Omega^{\nabla}$ as the two-sided ideal in $F Q^{\nabla}$ generated by $\Omega$ and the following elements:

(i) $u_{i p}^{-} w$, where $w: i \rightarrow j$ runs through all paths in 2 such that $w v \in \Omega$ for any path $v$ : $j \rightarrow p, p \in \boldsymbol{p}(\mathscr{Q}), j \in \mathscr{Q}_{0}$.

(ii) $\lambda_{1} u_{i p}^{-} w_{1}-\lambda_{2} u_{t p}^{-} w_{2}$, where $p \in \boldsymbol{p}(\mathscr{Q}), i, t, j \in \mathscr{Q}_{0}, \lambda_{1}, \lambda_{2} \in F$ and $w_{1}: i \rightarrow j, w_{2}: t \rightarrow j$ are paths in 2 such that

$$
u_{i p}-\lambda_{1} w_{1} v \in \Omega \text { and } \quad u_{t p}-\lambda_{2} w_{2} v \in \Omega
$$

for some nonzero path $v: j \rightarrow p$ in 2 (see Fig. 0). In particular $u_{i p}^{-} u_{i p}-u_{t p}^{-} u_{t p} \in \Omega$. The ideal $\Omega^{\nabla}$ is generated by $\Omega^{\nabla} \cap F \mathscr{Q}^{\nabla}$

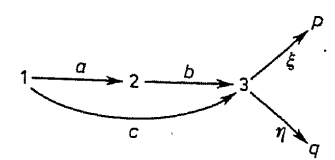

Fig. 1

Let us illustrate the definition by two examples.

EXAMPLE 2.17. Let $\mathscr{Q}$ be the quiver of Fig. 1 and $\Omega=(c \eta, a b \xi-c \xi)$. Then $R=F(\mathscr{Q}, \Omega)$ is a right two-peak algebra which is peak $\tilde{A}_{1}$-free, and $\mathscr{Q}^{\nabla}$ is shown in Fig. 2 and $\Omega^{\mathbf{v}}=(c \eta, a b \xi-c \xi, f c, e a b-e c)$, where $e=(c \xi)^{-}$and $f=(a b \eta)^{-}$. We note that $R \cong F\left(\mathscr{Q}^{\mathbf{\nabla}}, \Omega^{\mathbf{v}}\right)$

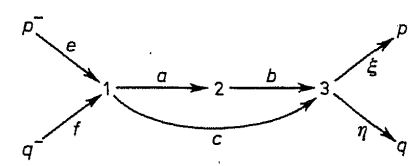

Fig. 2

EXAMPLE 2.18. Let $(\mathscr{Q}, \Omega)$ be the bound quiver obtained from $\left(\mathscr{Q}^{\prime}, \Omega^{\prime}\right)$ presented in Fig. 3, where

$\Omega^{\prime}=\left(h a c, a b, b a, f e-h d,(g e)^{-} g-(f e)^{-} f,(f e)^{-} h-(b d)^{-} b, u^{-} g,(g e)^{-} u\right.$,

$$
\left.(a c)^{-} d,(b d)^{-} c,(a c)^{-} a c-(b d)^{-} b d\right),
$$

by removing the vertices $p^{-}, q^{-}$and the edges starting from $p^{-}$and $q^{-}$. Note that $R=F(\mathscr{Q}, \Omega)$ is a right two-peak algebra, $R$ is not peak $\widetilde{\boldsymbol{A}}_{1}$-free, $\left(\mathscr{Q}^{\mathbf{v}}, \Omega^{\mathbf{\nabla}}\right)=\left(\mathscr{Q}^{\prime}, \Omega^{\prime}\right)$ and $R^{\boldsymbol{\nabla}} \cong F\left(\mathscr{2}^{\prime}, \Omega^{\prime}\right)$

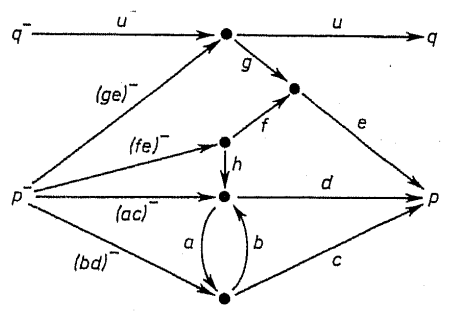

Fig. 3

Proposition 2.19. Let $R=F(2, \Omega)$ be a locally bounded right multipeak algebra which is peak $\tilde{A}_{1}$-free and nonsemisimple indecomposable. Then 
(a) $\mathscr{2}^{\nabla}$ and $\mathscr{Q}^{\nabla}$ are isomorphic to the quivers of $R^{\nabla}$ and $R^{\nabla}$, respectively.

(b) There are F-algebra isomorphisms

$$
\zeta: F\left(\mathscr{Q}^{\nabla}, \Omega^{\nabla}\right) \rightarrow R^{\nabla}, \quad \zeta^{\prime}: F\left(\mathscr{2}^{\nabla}, \Omega^{\nabla}\right) \rightarrow R^{\nabla},
$$

where $\left(\mathscr{Q}^{\nabla}, \Omega^{\nabla}\right)$ and $\left(\mathscr{Q}^{\nabla}, \Omega^{\nabla}\right)$ are the reflection forms of $(\mathscr{Q}, \Omega)$.

Proof. Let us define an algebra homomorphism $f: F, Q^{\mathbf{V}} \rightarrow R$ by taking for $\left.f\right|_{F 2}$ the natural epimorphism $F \mathscr{Q} \rightarrow R$ and by putting

$$
\begin{aligned}
& f\left(e_{p-}\right)=e_{p}^{-} \quad \text { if } p \in p(2), \\
& f\left(u_{i p}^{-}\right)=\bar{u}_{i p}^{*} \quad \text { if } u_{i p} \text { is the fixed edge maximal modulo } \Omega,
\end{aligned}
$$

where $\bar{u}_{i p}^{*} \in e_{p}^{-} R^{\nabla} e_{i}=D\left(e_{i} R e_{p}\right)$ is given by $\bar{u}_{i p}^{*}\left(u_{i p}\right)=1$.

Since $R$ is locally bounded, nonsemisimple and indecomposable as an algebra, given $p \in p(2)$ there is $i \in \mathscr{Q}_{0}-p(2)$ such that $e_{i} R e_{p} \neq 0$ and $e_{i} R e_{p}$ contains the coset $\bar{u}_{i p}$ of the maximal path $u_{i p}$ modulo $\Omega$. Since $R$ is peak $\tilde{A}_{1}$-free, according to Lemma 2.15 the map

$$
\mu_{p i p}: e_{p}^{-} R^{\mathbf{v}} e_{i} \otimes e_{i} R e_{p} \rightarrow e_{p}^{-} R^{\mathbf{v}} e_{p}
$$

is bijective and therefore $e_{p}^{-} R^{\nabla} e_{p}=\bar{u}_{i p}^{*} R^{\mathbf{v}} \subseteq \operatorname{Im} f$. In order to prove that $f$ is surjective it is sufficient to show that $e_{p}^{-} R^{\nabla} e_{i} \subseteq \operatorname{Im} f$ for all $i \in \mathscr{Q}_{0}$. For this purpose suppose that $e_{p}^{-} R^{\nabla} e_{i}=D\left(e_{i} R e_{p}\right) \neq 0$. Hence $e_{i} R e_{p} \neq 0$ and by our assumption there exists $j \in \mathscr{Q}_{0}$ such that $u_{j p}$ is maximal modulo $\Omega$ and $u_{j p}-\lambda v u_{i p} \in \Omega$ for some $\lambda \in F$ and a path $v: j \rightarrow i$. It follows from Lemma 2.15 that $\bar{u}_{j p}^{*} \bar{v} \in e_{p}^{-} R^{\nabla} e_{i}$ is nonzero since $\tilde{c}_{p j i}\left(\bar{u}_{p}^{*} \otimes v\right)\left(\lambda \bar{u}_{i p}\right)$ $=\bar{u}_{j p}^{*}\left(\bar{u}_{j p}\right)=1$. Hence $e_{p}^{-} R^{\mathbf{\nabla}} e_{i}=\bar{u}_{j p}^{*} R^{\mathbf{\nabla}} \subseteq \operatorname{Im} f$ and therefore $f$ is surjective. Moreover, the considerations above show that

$$
e_{p}^{-} R^{\mathbf{\nabla}} e_{j} / e_{p}^{-} J\left(R^{\mathbf{\nabla}}\right)^{2} e_{j}=\bar{u}_{j p}^{*} F \quad \text { if } u_{j p} \text { is maximal modulo } \Omega
$$

$=0 \quad$ otherwise.

It follows that the quiver of $R^{\nabla}$ is isomorphic to $2^{\nabla}$, which proves (a).

Applying Lemma 2.15(b) we easily check that $\Omega^{\nabla} \subseteq \operatorname{Ker} f$ and therefore $f$ induces an algebra epimorphism $\zeta: F\left(\mathscr{Q}^{\mathbf{V}}, \Omega^{\mathbf{v}}\right) \rightarrow R^{\mathbf{V}}$. In order to show that $\zeta$ is injective it is sufficient to check that the induced surjections

$$
\zeta_{p i}: e_{p-} F\left(\mathscr{Q}^{\mathbf{v}}, \Omega^{\mathbf{v}}\right) e_{i} \rightarrow e_{p}^{-} R^{\mathbf{v}} e_{i}
$$

are injective for all $p \in \boldsymbol{p ( 2 )}$ and $i \in \mathscr{Q}_{0}$. Since a simple analysis shows that $\operatorname{dim}_{F}\left(e_{p}-F\left(Q^{\nabla}, \Omega^{\nabla}\right) e_{i}\right) \leqslant 1$ it remains to show that if $e_{p}^{-} R^{\nabla} e_{i}=0$ then $e_{p--} F\left(\mathscr{Q}^{\mathbf{\nabla}}, \Omega^{\mathbf{\nabla}}\right) e_{i}=0$. Indeed, let $u: p^{-} \rightarrow i$ be a nonzero path in $\mathscr{Q}^{\mathbf{V}}$. Then $u=u_{i p}^{-} w$ for some path $w: i \rightarrow j$ in 2 . Since $e_{p}^{-} R^{\nabla} e_{i} \cong D\left(e_{i} R e_{p}\right)=0$ we get $e_{i} R e_{p}=0$ and therefore $w v \in \Omega$ for every path $v: j \rightarrow p$ in 2 . It follows from Definition 2.16(i) that $u=u_{i p} w \in \Omega^{\nabla}$. This shows that $e_{p}-F\left(Q^{\nabla}, \Omega^{\nabla}\right) e_{i}=0$ and proves that $\zeta$ is an isomorphism. Since the isomorphism $\zeta^{\prime}$ is the restriction of $\zeta$ to $F\left(\mathscr{Q}^{\nabla}, \Omega^{\nabla}\right)$ the proof is complete.

It would be interesting to describe the bound quiver of $F(\mathscr{Q}, \Omega)^{\nabla}$ and of $F(\mathscr{Q}, \Omega)^{\nabla}$ in terms of $(2, \Omega)$ in the general situation.
Note that the algebra $R$ in Example 2.18 is not peak $\tilde{A}_{1}$-free while $R^{\mathbf{\nabla}} \cong F\left(Q^{\mathbf{v}}, \Omega^{\mathbf{v}}\right)$. However, this fact does not hold in general because of the following example.



Fig. 4

ExAmple 2.20. Let $R=F(\mathscr{2}, \Omega)$, where $\mathscr{2}$ is the quiver of Fig. 4 and $\Omega=(h b, a u+c w-b v)$. Applying the same type of argument as in the proof of Proposition 2.19 one can show that $R^{\mathbf{\nabla}} \cong F\left(\mathscr{Q}^{\prime}, \Omega^{\prime}\right)$, where $\mathscr{Q}^{\prime}$ is shown in Fig. 5 and $\Omega^{\prime}=\left(\Omega,(c w)^{*} a,(c w)^{*} c+(h a u)^{*} h c\right)$. Note that $\mathscr{Q}^{\mathbf{V}}$ is not isomorphic to $\mathscr{Q}^{\prime}$

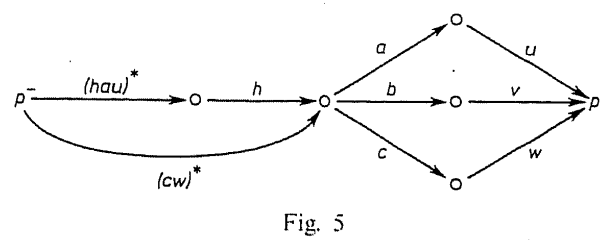

Definition 2.21. Let $(2, \Omega)$ be a right multipeak bound quiver. The reflection dual bound quiver of $(2, \Omega)$ is the bound quiver $\left(2^{\bullet}, \Omega^{\bullet}\right)=\left(\mathscr{Q}^{\nabla}, \Omega^{\nabla}\right)^{\mathrm{op}}$.

As an immediate consequence of Proposition 2.19 we get

CoRollaRy 2.22. If $(\mathscr{Q}, \Omega)$ is a right multipeak bound quiver such that $F(\mathscr{Q}, \Omega)$ is $\tilde{A}_{1}$-free then $\left(\mathscr{Q}^{\bullet}, \Omega^{\bullet}\right)$ is a right multipeak bound quiver and there is an F-algebra isomorphism $(F(\mathscr{Q}, \Omega))^{\bullet} \cong F\left(\mathscr{Q}^{\bullet}, \Omega^{\bullet}\right)$.

3. Main results. Throughout this section $(\mathscr{Q}, \Omega)$ denotes a connected right multipeak bound quiver and

$$
R=F(2, \Omega)=\bigoplus_{i \in \mathscr{Q}_{0}} e_{i} R,
$$

where $e_{i}: i \rightarrow i$ is the trivial path in 2 . Moreover, we suppose that 2 is directed, i.e. the relation

$$
i \prec j \Leftrightarrow \text { there is a nonzero path } i \rightarrow j \text { in } 2
$$

is a partial order in $\mathscr{Q}_{0}$ [17]. Given $t \in \mathscr{Q}_{0}$ we denote by $t^{\nabla}$ (resp. by $t^{\Delta}$ ) the full bound subquiver of $(2, \Omega)$ consisting of vertices $s \in \mathscr{Q}_{0}$ such that there is a path $s \rightarrow t$ (resp. $t \rightarrow s$ ) in 2 which does not belong to $\Omega$. The idea of the splittting decomposition of posets [15] and of right peak rings [11] is extended as follows.

Following [11; Section 4] we say that subposets $\mathscr{Q}_{0}^{\prime}, \mathscr{C}_{0}$ and $\mathscr{Q}_{0}^{\prime \prime}$ of $\mathscr{Q}_{0}$ form 
a triangular decomposition of the bound quiver $(2, \Omega)$ if

$$
\mathscr{Q}_{0}=\mathscr{Q}_{0}^{\prime}+\mathscr{C}_{0}+\mathscr{Q}_{0}^{\prime \prime}
$$

is a disjoint union of subposets, $\mathscr{Q}_{0}^{\prime}$ and $\mathscr{Q}_{0}^{\prime \prime}$ are nonempty and

(i) there are no relations $j^{\prime \prime} \prec j^{\prime}, j^{\prime \prime} \prec c, c \prec j^{\prime}$ with $j^{\prime} \in \mathscr{Q}_{0}^{\prime}, c \in \mathscr{C}_{0}$ and $j^{\prime \prime} \in \mathscr{Q}_{0}^{\prime \prime}$,

(ii) $\mathscr{C}_{0} \subseteq \mathscr{Q}_{0}-p(\mathscr{2})$ and $\left(\mathscr{C}+\mathscr{Q}^{\prime \prime}, \Omega \mid\right)$ is a right multipeak bound quiver with the set peaks $p\left(\mathscr{C}+\mathscr{Q}^{\prime \prime}\right)=\mathscr{Q}_{0}^{\prime \prime} \cap p(\mathscr{Q})$, where $\Omega|=\Omega|_{\mathscr{b}}+\mathscr{Q}^{\prime \prime}$.

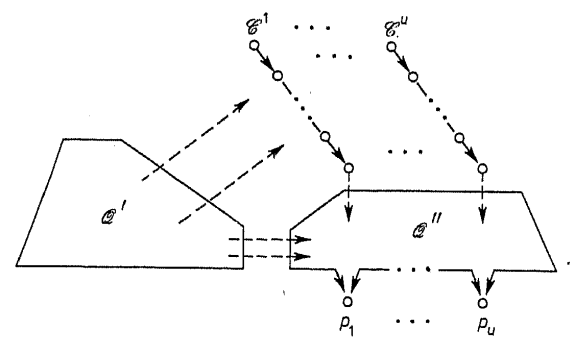

Fig. 6

DeFinition 3.3. The triangular decomposition (3.2) is called a splitting decomposition of $(\mathscr{Q}, \Omega)$ if there exist a nonempty set

$$
\mathscr{P}=\left\{p_{1}, \ldots, p_{u}\right\} \subseteq p(\mathscr{Q}) \cap \mathscr{Q}_{0}^{\prime \prime}, \quad p_{j} \neq p_{i} \text { for } j \neq i,
$$

and a disjoint union poset decomposition (see Fig. 6)

$$
\mathscr{C}_{0}=\dot{\mathscr{C}}_{0}^{1}+\ldots+\mathscr{C}_{0}^{u}
$$

where $\mathscr{C}_{0}^{1}, \ldots, \mathscr{C}_{0}^{u}$ are pairwise incomparable chains such that

(a) $\left(\mathscr{Q}^{\prime}+\mathscr{C}+\mathscr{P}, \Omega \mid\right)$ is a connected right multipeak bound quiver satisfying $p\left(\mathscr{Q}^{\prime}+\mathscr{C}+\mathscr{P}\right)=\boldsymbol{p}(\mathscr{2}) \cap \mathscr{Q}_{0}^{\prime} \cup \mathscr{P}$

(b) The full bound subquiver $\hat{\mathscr{C}}=\mathscr{C} \cup \mathscr{P}$ of $(\mathscr{Q}, \Omega)$ consisting of vertices $\mathscr{C}_{0} \cup \mathscr{P}$ is a poset disjoint union

$$
\hat{\mathscr{C}}=\mathscr{C} \cup \mathscr{P}=\hat{\mathscr{C}}^{1}+\ldots+\hat{\mathscr{C}}^{u}
$$

of pairwise unrelated chains

$$
\mathscr{C}^{i}: c_{1}^{i} \rightarrow c_{2}^{i} \rightarrow \ldots \rightarrow c_{t_{i}}^{i} \rightarrow c_{t_{i}+1}^{i}=p_{i}
$$

where $t_{i} \geqslant 0$. The restriction of $\Omega$ to $\hat{\mathscr{C}}$ is empty.

(c) For any $i \in \mathscr{Q}_{0}^{\prime}$ and $j \in \mathscr{Q}_{0}^{\prime \prime}$ we have

$$
d(i, j)=\sum_{p \in \mathscr{P}} d(i, p) d(j, p)
$$

where $d(s, t)=\operatorname{dim}_{F}\left(e_{s} R e_{t}\right)$. Moreover, $d(c, q)=0$ for all $c \in \mathscr{C}$ and $q \in p(\mathscr{Q})-\mathscr{P}$
It follows that $(2, \Omega)$ has the form of Fig. 6.

Note also that $d(i, q)=0$ for all $i \in \mathscr{Q}_{0}^{\prime}, q \in \boldsymbol{p}\left(\mathscr{Q}^{\prime \prime}\right)-\mathscr{P}$, for any $p \in \mathscr{P}$ there is $i \in \mathscr{Q}_{0}^{\prime}$ such that $d(i, p) \neq 0$, and $d(i, j) \neq 0$ iff there is $p \in \mathscr{P}$ such that $d(i, p) \neq 0$ and $d(j, p) \neq 0$. In general the sets $\mathscr{Q}_{0}^{\prime}-p(\mathscr{Q})$ and $p\left(\mathscr{Q}^{\prime \prime}\right)-\mathscr{P}$ are not empty.

Our main motivation for the definition above is Theorem 3.10 below which shows how the category $\bmod _{\mathrm{sp}}(R)$ can be obtained by glueing $\bmod _{\mathrm{sp}} F\left(\mathscr{2}^{\prime}+\hat{\mathscr{C}}, \Omega \mid\right)$ and $\bmod _{\mathrm{sp}} F\left(\mathscr{C}+\mathscr{Q}^{\prime \prime}, \Omega \mid\right)$ if the decomposition (3.2) is splitting. This result is of "recollements" character.

The reader is referred to Section 4 for typical examples of splitting decomposition and its applications.

Remark $3.3^{\prime}$. Suppose that $R=F(\mathscr{Q}, \Omega)$. It is easy to check that $R^{\bullet} \cong F\left(\mathscr{Q}^{\bullet}, \Omega^{\bullet}\right)$ and

$$
\mathscr{2}_{0}^{\bullet}=\left(\mathscr{Q}_{0}-p(\mathscr{Q})\right) \cup p(\mathscr{2})^{-}, \quad \mathscr{Q}^{\bullet}-p(\mathscr{2})^{-} \cong(\mathscr{Q}-p(\mathscr{2}))^{\mathrm{op}}
$$

where $p(2)^{-}=\left\{p^{-}, p \in p(\mathscr{2})\right\}$. One can check that if (3.2) is a splitting decomposition of $(\mathscr{Q}, \Omega)$ then $\left(\mathscr{Q}^{\bullet}, \Omega^{\bullet}\right)$ admits a splitting decomposition given by the set $\mathscr{P}^{-}$of peaks and by

$$
\mathscr{2}_{0}^{\bullet}=\dot{2}_{0}^{\prime \prime}+\mathscr{C}_{0}^{\mathrm{op}}+\bullet_{\mathscr{Q}_{0}^{\prime}}
$$

where $\mathscr{Q}_{0}^{\prime \prime}=\left(\mathscr{Q}_{0}^{\prime \prime}-p\left(\mathscr{Q}^{\prime \prime}\right)\right) \cup\left(\boldsymbol{p}\left(\mathscr{Q}^{\prime \prime}\right)-\mathscr{P}\right)^{-}, \bullet_{\mathscr{Q}_{0}^{\prime}}=\left(\mathscr{Q}_{0}^{\prime}-\boldsymbol{p}\left(\mathscr{Q}^{\prime}\right)\right) \cup p\left(\mathscr{Q}^{\prime}\right)^{-} \cup \mathscr{P}^{-}$. We note that

$$
\operatorname{dim}_{F}\left(e_{j} R^{\bullet} e_{i}\right)=d(i, j) \quad \text { for } i \in \mathscr{Q}_{0}^{\prime}, j \notin p(\mathscr{Q}),
$$$$
\operatorname{dim}\left(e_{i} R^{\bullet} e_{p}^{-}\right)=d(i, p) \quad \text { for } p \in \boldsymbol{p}(\mathscr{2}) \text {. }
$$

Given a splitting decomposition (3.2) we consider

$$
\hat{S}=\hat{\eta} R \hat{\eta}=F\left(\mathscr{Q}^{\prime}+\hat{\mathscr{C}}, \Omega \mid\right) \quad \text { and } \quad R=\left(\begin{array}{cc}
S & { }_{S} M_{T} \\
0 & T
\end{array}\right)
$$

where $S=\eta R \eta=F\left(\mathscr{Q}^{\prime}, \Omega \mid\right), M=\eta R \xi, T=\xi R \xi=F\left(\mathscr{C}+\mathscr{2}^{\prime \prime}, \Omega \mid\right)$ and

$$
\eta=\sum_{i \in \mathscr{Q}_{0}} e_{i}, \quad \xi=\sum_{j \in \mathscr{Q}_{0}-\mathscr{2}_{0}} e_{j}, \quad \hat{\eta}=e_{p_{1}}+\ldots+e_{p_{u}}+\sum_{j \in \mathscr{Q}_{j} \cup \mathscr{Q}} e_{j}
$$

Our splitting theorem will be formulated in terms of the functors (see (2.10))

$$
\bmod _{\mathrm{sp}}(\hat{S}) \underset{\boldsymbol{L}_{\hat{\eta}}, \boldsymbol{r}_{\hat{\eta}}}{\rightleftarrows} \bmod _{\mathrm{sp}}(R) \underset{r_{\xi}}{\stackrel{T_{\xi}}{\leftrightarrows}} \bmod _{\mathrm{sp}}(T) \text {. }
$$

Let us start with three technical lemmas.

Lemma 3.7. Let $A=F\left(\mathscr{Q}^{\prime \prime}, \Omega \mid\right)=f R f$, where $f=\sum_{j \in \mathscr{Q}^{\prime \prime}} e_{j}$. A module $X$ in $\bmod _{\mathrm{sp}}(R)$ belongs to $\operatorname{Im} L_{\hat{\eta}}$ if and only if $X f^{\prime} \cong \bigoplus_{p \in \mathscr{P}} E_{A}\left(e_{p} A\right)^{r_{p}}$ for some $r_{p} \geqslant 0$. In this case $X \cong L_{\hat{\eta}} r_{\eta}(X) \cong T_{\hat{\eta}} r_{\eta}(X)$ 
Proof. Suppose that $X \cong L_{\hat{\eta}}(Y)$ with $Y$ in $\bmod _{\text {sp }}(\hat{S})$ and let $g=e_{p_{1}}+\ldots+e_{p_{u}}$. Since $G=g A g$ is a product of division rings and the functor $L_{g}: \bmod (G) \rightarrow \bmod (A)$ is right adjoint to the restriction functor $r_{g}, L_{g}(V)$ is injective and $\operatorname{soc} L_{g}(V) \cong L_{g}(V) g \cong V$ for any $V$ in $\bmod (G)$. Since obviously $L_{\hat{\eta}}(Y) f=L_{g}(Y g), X f$ is $A$-injective as required.

In order to prove the converse we note that since $\boldsymbol{L}_{\hat{\eta}}$ is right adjoint to $\boldsymbol{r}_{\hat{\eta}}$ there is a natural $R$-homomorphism $h: X \rightarrow L_{\hat{\eta}} r_{\hat{\eta}}(X)$ such that $\boldsymbol{r}_{\hat{\eta}}(h)=$ id and the induced map $\operatorname{soc}(X) \rightarrow \operatorname{soc}\left(L_{\hat{\eta}} r_{\hat{\eta}}(X)\right)$ is bijective. By the "if" part of the lemma $L_{\hat{\eta}} r_{\hat{\eta}}(X) f$ is $A$-injective and therefore $h$ restricted to $X f$ is an isomorphism. It follows that $h$ is bijective. Since analogously the natural map $\boldsymbol{T}_{\hat{\eta}} \boldsymbol{r}_{\hat{\eta}}(X) \rightarrow X$ is also bijective the proof is complete.

LEMMA 3.8. (a) Given $i=1, \ldots, u$ the T-modules

$$
H_{0}^{i}:=E_{T}\left(e_{p_{i}} T\right)=D_{T}^{\bullet}\left(e_{p_{i}}^{-} T^{\bullet}\right) \text { and } H_{j}^{i}:=D_{T}^{\bullet}\left(e_{c_{j}^{i}} T^{\bullet}\right) \text { for } j=1, \ldots, t_{i}
$$

(see (3.3)) are indecomposable hereditary sp-injective in $\bmod _{\mathrm{sp}}(T)$ such that $H_{j}^{i} f \cong E_{A}\left(e_{p_{t}} A\right)$, $\left.H_{j}^{i}\right|_{q_{t}}=0$ for $t \neq i$ and

$$
H_{j \mid \hat{\mathscr{E}}^{i}}^{i}=(0 \rightarrow 0 \rightarrow \ldots \rightarrow 0 \rightarrow F \rightarrow F \rightarrow \ldots \rightarrow F) \quad(j \text { zeros })
$$

for all $i=1, \ldots, u, j \leqslant t_{i}+1$. The natural embeddings

$$
H_{t_{i}}^{i} \subseteq \ldots \subseteq H_{1}^{i} \subseteq H_{0}^{i}=E_{T}\left(e_{p_{i}} T\right)
$$

are irreducible in $\bmod _{\text {sp }}(T)$. If $\operatorname{Hom}_{T}\left(H_{j}^{i}, Z\right) \neq 0$ and $Z$ is in $\operatorname{ind}_{\text {sp }}(T)$ then $Z \cong H_{r}^{i}$ for some $0 \leqslant r \leqslant j$. Moreover, $\operatorname{Hom}_{T}\left(H_{r}^{i}, H_{s}^{j}\right)=0$ for all $r, s$ and $i \neq j$.

(b) The $\hat{S}$-modules $P_{j}^{i}=e_{c_{j}^{i}} \hat{S}, j=1, \ldots, t_{i}, t_{i}+1$, are hereditary projective and the natural embeddings

$$
e_{p_{i}} \hat{S}=P_{t_{i}+1}^{i} \subseteq P_{t_{i}}^{i} \subseteq \ldots \subseteq P_{1}^{i}
$$

are irreducible in $\bmod _{\mathrm{sp}}(\hat{S})$.

$$
\text { (c) } \quad \boldsymbol{L}_{\hat{\eta}}\left(P_{j}^{i}\right) \cong \boldsymbol{T}_{\xi}\left(H_{j-1}^{i}\right) \quad \text { for } j=1, \ldots, t_{i}+1, i=1, \ldots, u \text {. }
$$

Proof. Applying the same type of argument as in the proof of Proposition 2.19 we show that the support of the module $X=e_{c_{j}^{i}} T^{\bullet}$ is contained in $\mathscr{S}: p_{i}^{-} \leftarrow c_{1}^{i} \leftarrow \ldots \leftarrow c_{t_{i}}^{i}$ and we have

$$
\left.X\right|_{\mathscr{S}}=\left(F^{\mathrm{op}} \leftarrow \ldots \leftarrow F_{j}^{\mathrm{op}} \leftarrow \underset{j+1}{0} \leftarrow \ldots \leftarrow 0\right)
$$

Then $X$ is hereditary projective and Proposition 2.5(c) together with the reflection duality $D_{T}^{\bullet}$ yields (a).

(b) Since obviously $\operatorname{supp}\left(P_{j}^{i}\right) \subseteq \hat{\mathscr{C}}^{i}$, (b) follows.

(c) It follows from the definitions that $\operatorname{supp}\left(L_{\hat{\eta}}\left(P_{j}^{i}\right)\right), \operatorname{supp}\left(T_{\xi}\left(H_{j-1}^{i}\right)\right) \subseteq \mathscr{C}^{l} \cup \mathscr{Q}^{\prime \prime}$, $\left.\left.\left.\boldsymbol{T}_{\xi}\left(H_{j-1}^{i}\right)\right|_{q_{i}} \cong H_{j-1}^{i}\right|_{q_{i}} \cong P_{j}^{i}\right|_{\xi_{i}}$ and $\boldsymbol{T}_{\xi}\left(H_{j-1}^{i}\right) f \cong E_{A}\left(e_{p_{i}} A\right)$ (in the notation of Lemma 3.7). Then Lemma 3.7 yields $T_{\xi}\left(H_{j-1}^{i}\right) \cong L_{\hat{\eta}} r_{\hat{\eta}} T_{\xi}\left(H_{j-1}^{i}\right) \cong L_{\hat{\eta}}\left(P_{j}^{i}\right)$ and (c) follows.

LEMMA 3.9. (a) Given $i \in \mathscr{Q}_{0}^{\prime}$ the restriction of $e_{i} R$ to $\mathscr{C}+\mathscr{Q}^{\prime \prime}$ is either zero or is isomor- phic to a direct sum of copies of $H_{j}^{i}$ for some indices $i$ and $j$. The module $M_{T}$ in (3.4) is hereditary sp-injective and $M_{T}$ is isomorphic to a direct sum of copies of $H_{j}^{i}, i=1, \ldots, u$, $j=0, \ldots, t_{i}$ (comp. [28]).

(b) The functors $T_{\xi}, L_{\eta}$ are exact and

$$
\begin{aligned}
& \boldsymbol{T}_{\xi}\left(c_{i} T\right) \cong e_{i} R \quad \text { for } i \in \mathscr{Q}_{0}-\mathscr{Q}_{0}^{\prime}, \quad \boldsymbol{L}_{\eta}\left(e_{j} \hat{S}\right) \cong e_{j} R \text { for } j \in \mathscr{Q}_{0}^{\prime}, \\
& T_{\xi}\left(Q_{T^{(j)}}\right) \cong Q^{(j)} \quad \text { for } j \in \mathscr{Q}_{0}^{\prime \prime}, \quad \boldsymbol{L}_{\hat{h}}\left(Q_{S}^{(j)}\right) \cong Q^{(j)} \quad \text { for } j \in \mathscr{Q}_{0}^{\prime}+\hat{\mathscr{C}} .
\end{aligned}
$$

Proof. (a) Lét $i \in \mathscr{Q}_{0}^{\prime}$. Since $e_{i} R$ is socle projective, Definition 3.3(c) yields

$$
\operatorname{soc}\left(e_{i} R f\right)=\bigoplus_{p \in \mathscr{P}}\left(e_{p} A\right)^{d(i, p)}
$$

where $f$ and $A$ are as in Lemma 3.7. It follows that there is an $A$-monomorphism

$$
h: e_{i} R f \rightarrow \underset{p \in \mathscr{P}}{\bigoplus} E_{A}\left(e_{p} A\right)^{d(i, p)} .
$$

Since $\operatorname{dim}_{F} E_{A}\left(e_{p} A\right) e_{j}=d(j, p)$, the splitting assumption (c) yields

$$
\begin{aligned}
\operatorname{dim}_{F}\left(e_{i} R f e_{j}\right)=d(i, j) & =\sum_{p \in I^{P}} d(i, p) d(j, p) \\
& =\operatorname{dim}_{F}\left(\bigoplus_{p \in\{;} E_{A}\left(e_{p} A\right)^{d(i, p)}\right) e_{j}
\end{aligned}
$$

for any $j \in \mathscr{Q}_{0}^{\prime \prime}$ and therefore $h$ is bijective.

In order to finish the proof we consider the functors

$$
\bmod _{\mathrm{sp}}(\Lambda) \frac{\underline{x}_{w}}{\overbrace{w}} \bmod _{\mathrm{sp}}(T)
$$

where $\Lambda=w T w$ and $w=\sum_{j e} e_{j}$. By our assumptions $\Lambda \cong F \hat{\mathscr{C}}^{1} \times \ldots \times F \hat{\mathscr{C}}^{u}$ and $\bmod _{\mathrm{sp}}\left(F \hat{\mathscr{C}}^{\jmath}\right) \cong \mathscr{C}^{j}$-sp, where $\mathscr{\mathscr { C }}^{1}, \ldots, \mathscr{\mathscr { C }}^{u}$ are the chains in Definition 3.3. Hence if $X$ is in $\operatorname{ind}_{\mathrm{sp}}(\Lambda)$ then $\operatorname{supp}(X) \subseteq \hat{C}^{r}$ for some $r \leqslant u$ and

$$
\left.X\right|_{\mathbb{R}^{\prime}}=(0 \rightarrow 0 \rightarrow \ldots \rightarrow 0 \rightarrow F \rightarrow F \rightarrow \ldots \rightarrow F) \quad(j \text { zeros }) .
$$

Since we know from Lemma 3.7 that $L_{w}(X) f \cong L_{\hat{\eta}}(X) f \cong E_{A}\left(e_{p_{r}} A\right)$, Lemma 3.8(a) yields $\boldsymbol{L}_{w}(X) \cong H_{j}^{r}$ and therefore in order to prove that the restriction $Y$ of $e_{i} R$ to $\mathscr{C}+\mathscr{Q}^{\prime \prime}$ is a direct sum of copies of $H_{s}^{t}$ it is sufficient to show that $Y$ is in $\operatorname{Im} L_{w}$. For this purpose we note that since $Y f^{\prime} \cong e_{l} R f, Y \eta=0$ and the map $h$ is an $A$-isomorphism, according to Lemma 3.7 we have $Y \cong L_{\hat{\eta}} r_{\hat{\eta}}(Y) \cong L_{w} r_{w}(Y)$ as required.

Since

$$
M_{T}=\eta R \xi=\sum_{l \in Q_{0}^{\prime}} \sum_{G \in Q_{0}-\mathscr{L}_{0}^{\prime}} e_{i} R e_{j}=\bigoplus_{l \in Q_{0}^{\prime}} r_{\xi}\left(e_{l} R\right)
$$

and since it follows from the fact proved above that $\boldsymbol{r}_{\xi}\left(e_{l} R\right)$ is either zero or a direct sum of copies of $H_{j}^{r}$, (a) follows in view of Lemma 3.8 .

(b) First we note that $\boldsymbol{T}_{4}$ is the embedding of $\bmod _{\mathrm{sp}}(T)$ in $\bmod _{\mathrm{sp}}(R)$ induced by the natural epimorphism $R \rightarrow T$ derived from the triangular form (3.4) of $R$. The functor 
$L_{\hat{\eta}}$ is left exact by Proposition 2.11(a). Therefore from Lemma 3.7 and from $\boldsymbol{r}_{\hat{\eta}} L_{\hat{\eta}} \cong$ id we easily conclude that $L_{\hat{\eta}}$ is exact.

The first isomorphism in (b) is obvious. In order to prove the second one we fix $j \in \mathscr{Q}_{0}^{\prime \prime}-\boldsymbol{p}(2)$ and recall from $(2,7)$ that $Q^{(j)} \cong \nabla_{+} D\left(e_{j} R^{\bullet}\right) \cong D \nabla_{-}\left(e_{j} R^{\bullet}\right)$. By Proposition 2.5 (c) there is an exact sequence

$$
0 \rightarrow e_{j} R^{\bullet} \stackrel{h}{\rightarrow} E_{\tilde{R}^{\bullet}}\left(e_{j} R^{\bullet}\right) \rightarrow \nabla_{-}\left(e_{j} R^{\bullet}\right) \rightarrow 0
$$

in $\bmod \left(\hat{R}^{\bullet}\right)$, where $\hat{R}^{\bullet}=\left(R^{\nabla}\right)^{\text {op }}$ and $\bmod _{\mathrm{sp}}\left(R^{\bullet}\right)$ is considered as a full subcategory of $\bmod \left(\hat{R}^{\bullet}\right)$ via the natural epimorphism $\hat{R}^{\bullet} \rightarrow R^{\bullet}$. Consider the idempotents

$$
\eta_{1}=\sum_{i \in \epsilon^{\bullet} \mathscr{Q}_{0}^{\prime}} e_{i}, \quad \xi_{1}=\sum_{j \in \epsilon^{\bullet} \mathscr{Q}_{0}^{\prime \prime}+\mathscr{E}+\mathscr{P}^{-}} e_{j}
$$

in $R^{\bullet}$ and the idempotent

$$
\xi_{2}=\xi_{1}+\sum_{p \in p\left(\mathscr{Q}^{\prime \prime}\right)} e_{p}
$$

in $R^{\bullet}$, where we put $e_{p--}=e_{p}^{-}$for $p \in p(\mathscr{2})$ and ${ }^{\bullet} 2^{\prime}, \bullet^{\prime \prime}$ are as in Remark $3.3^{\prime}$. It is easy to see that $T^{\bullet} \cong \xi_{1} \hat{R}^{\bullet} \xi_{1}$ and $\hat{T}^{\bullet} \cong \xi_{2} \hat{R}^{\bullet} \xi_{2}$. A simple analysis shows that $E_{T^{\bullet}}\left(e_{j} T^{\bullet}\right)$ $=E_{\hat{R}^{\bullet}}\left(e_{j} R^{\bullet}\right) \xi_{2}$ (the restriction of $E_{\hat{R}^{\bullet}}\left(e_{j} R^{\bullet}\right)$ to $\left(\mathscr{P}^{-}+\mathscr{C}+\mathscr{Q}^{\prime \prime}\right)^{\text {op }}$ ). By Remark $3.3^{\prime}$ the decomposition $\mathscr{2}^{\bullet}=\bullet^{\bullet} \mathscr{2}^{\prime}+\mathscr{C}^{\text {op }}+\mathscr{2}^{\prime \prime}$ induces a splitting decomposition of $\left(\mathscr{Q}^{\bullet}, \Omega^{\bullet}\right)$. Then applying (a) to $R^{\bullet}$ we conclude that the restriction $e_{j} R^{\bullet} \eta_{1}$ of $e_{j} R^{\bullet}$ to ${ }^{\bullet} \mathscr{Q}_{0}^{\prime}$ is an injective module and therefore the restriction $e_{j} R^{\bullet} \eta_{1} \rightarrow E_{R^{\bullet}}\left(e_{j} R^{\bullet}\right) \eta_{1}$ of the map $h$ to $e_{j} R^{\bullet} \eta_{1}$ is an isomorphism. It follows that Cokerh is a $T^{\bullet}$-module and according to Proposition 2.5(c) and remarks above the sequence $(*)$ induces a commutative diagram

$$
\begin{aligned}
& 0 \rightarrow e_{j} R^{\bullet} \xi_{2} \rightarrow E_{R^{\bullet}}\left(e_{j} R^{\bullet}\right) \xi_{2} \rightarrow \nabla_{-}\left(e_{j} R^{\bullet}\right) \rightarrow 0 \\
& \underset{0 \rightarrow e_{j} T^{\bullet}}{\stackrel{\downarrow}{\rightarrow}} \underset{E_{T^{\bullet}}\left(e_{j} T^{\bullet}\right)}{\downarrow} \rightarrow \nabla_{-}\left(e_{j} T^{\bullet}\right) \rightarrow 0
\end{aligned}
$$

of $\hat{T}^{\bullet}$-modules. Since $\boldsymbol{T}_{\boldsymbol{\xi}}$ is a natural embedding we have

$$
T_{\xi}\left(Q_{T}^{(j)}\right)=Q_{T}^{(j)} \cong D \nabla_{-}\left(e_{j} T^{\bullet}\right) \cong D \nabla_{-}\left(e_{j} R^{\bullet}\right) \cong Q^{(j)}
$$

as required. For $j \in \boldsymbol{p}\left(\mathscr{Q}^{\prime \prime}\right)$ the proof is similar.

In order to establish the right hand isomorphisms in (b) we note that according to (a) and Lemma 3.8 the restriction of $e_{j} R$ to $2^{\prime \prime}$ is isomorphic to a direct sum of copies of the $A$-modules $E_{A}\left(e_{p} A\right), p \in \mathscr{P}$. Then by Lemma 3.7 we get

$$
e_{j} R \cong L_{\hat{\eta}} \boldsymbol{r}_{\hat{\eta}}\left(e_{j} R\right) \cong L_{\hat{\eta}}\left(e_{j} R \hat{\eta}\right) \cong L_{\hat{\eta}}\left(e_{j} \hat{S}\right) \text {. }
$$

Since the remaining isomorphism in (b) can be established in a similar way the proof is complete.

Now we are able to prove our multipeak splitting theorem.

THEOREM 3.10. Suppose that $R=F(2, \Omega)$ is a right multipeak algebra (3.1) where $F$ is a division ring and $(2, \Omega)$ is a bound quiver with a splitting decomposition (3.2). In the notation of (3.6) we have: (a) If $X$ is in $\operatorname{ind}_{s p}(R)$ then either $X \in \operatorname{Im} T_{\xi}$ or $X \in \operatorname{Im} L_{\hat{\eta}}$. Moreover, $X \in \operatorname{Im} T_{\xi} \cap \operatorname{Im} \boldsymbol{L}_{\hat{\eta}}$ if and only if $X \cong L_{\hat{\eta}}\left(P_{j}^{i}\right) \cong T_{\xi}\left(H_{j-1}^{l}\right)$ for some $i=1, \ldots, u, j=1, \ldots, t_{i}, t_{i+1}$ (see 3.8). If $Y$ is in $\operatorname{ind}_{\mathrm{sp}}(\hat{S}), Z$ is in $\operatorname{ind}_{\mathrm{sp}}(T)$ and $Y \eta \neq 0$ then $\operatorname{Hom}_{R}\left(L_{\hat{\eta}}(Y), T_{\xi}(Z)\right)=0$.

(b) The functors $\boldsymbol{L}_{\hat{\eta}}$ and $\boldsymbol{T}_{\xi}$ are full faithful exact and carry Auslander-Reiten sequences to Auslander-Reiten sequences. If $\mathfrak{X}$ is an Auslander-Reiten sequence in $\bmod _{\mathrm{sp}}(R)$ and the starting term of $\mathfrak{X}$ is indecomposable then either $\mathfrak{X} \cong L_{\hat{\eta}}(\mathfrak{Y})$ or $\mathfrak{X} \cong T_{\xi}(3)$, where $\mathfrak{Y}$ and 3 are Auslander-Reiten sequences in $\bmod _{\mathrm{sp}}(\hat{S})$ and $\bmod _{\mathrm{sp}}(T)$, respectively.

Proof. Let $X=\left(X_{S}^{\prime}, X_{T}^{\prime \prime}, \varphi: X^{\prime} \otimes_{S} M_{T} \rightarrow X_{T}^{\prime \prime}\right)$ be a module in $\operatorname{ind}_{\mathrm{sp}}(R)$. It is clear that $X_{T}^{\prime \prime}=X \xi$ is in $\bmod _{\mathrm{sp}}(T)$. Assume $X_{T}^{\prime}=X \eta \neq 0$. If $\varphi \neq 0, Y$ is an indecomposable summand of $X_{T}^{\prime \prime}$ and $p: X^{\prime \prime} \rightarrow Y$ is the natural projection then $p \varphi \neq 0$ and therefore $\operatorname{Hom}_{T}\left(M_{T}, Y\right) \neq 0$. It follows from Lemma 3.8(a) that $Y \cong H_{j}^{r}$ for some $r$ and $j$. Consequently, either $\varphi=0$ and $X^{\prime \prime}=0$, or $X_{T}^{\prime \prime}$ is a direct sum of copies of $H_{j}^{r}$. Therefore $X f=X^{\prime \prime} f$ is injective because $H_{j}^{r} f \cong E_{A}\left(e_{p_{r}} A\right)$ in the notation of Lemma 3.7. It follows from Lemma 3.7 that $X \cong L_{\hat{\eta}} r_{\hat{\eta}}(X) \cong T_{\xi} r_{\xi}(X) \in \operatorname{Im} L_{\hat{\eta}}$ and $r_{\hat{\eta}}(X)$ is indecomposable. If $X_{S}^{\prime}=0$ then obviously $X \cong T_{\xi} \boldsymbol{r}_{\xi}(X) \in \operatorname{Im} \boldsymbol{T}_{\xi}$ and the first statement in (a) follows.

If $X \cong L_{\eta}(Y) \cong T_{\xi}(Z)$ then $X \eta=Y \eta=0$ and therefore $\operatorname{supp}(Y) \subseteq \hat{\mathscr{C}}^{r}$ for some $r=1, \ldots, u$ because of (3.3). Hence $Y \cong P_{j}^{r}$ for some $j$ and in view of Lemma 3.8(c) the second statement in (a) follows.

Now take $Y$ in $\operatorname{ind}_{s p}(\hat{S})$ such that $Y \eta \neq 0$, put $X \cong L_{\hat{\eta}}(Y)$ and suppose that there is a nonzero $h \in \operatorname{Hom}_{R}\left(X, T_{\xi} Z\right)$. It follows from the first part of the proof that $X \xi$ is a direct sum of copies of $H_{l}^{j}, j=1, \ldots, u, i=0, \ldots, t_{j}$. Now since $h \neq 0$ and $\left(T_{\xi} Z\right) \eta=0$, $r_{\xi}(h): X \xi \rightarrow\left(\boldsymbol{T}_{\xi} Z\right) \xi \cong Z$ is nonzero and we conclude from Lemma 3.8 that $Z \cong H_{j-1}^{i}$ for some $i$ and $j$, and $T_{\xi} Z \cong T_{\xi} H_{j-1}^{l} \cong L_{\eta} P_{j}^{i}$. Hence $\operatorname{Hom}_{R}\left(X, T_{\xi} Z\right) \cong \operatorname{Hom}_{R}\left(L_{\hat{\eta}} Y, L_{\hat{\eta}} P_{j}^{i}\right)$ $\cong \operatorname{Hom}_{S}\left(Y, P_{j}^{i}\right)=0$ because otherwise $Y \cong P_{l}^{i}$ for some $l \geqslant j($ Lemma $3.8(b))$ and therefore $Y_{\eta}=0$; a contradiction. This finishes the proof of (a).

(b) Suppose that $\mathfrak{Y}): 0 \rightarrow Y \stackrel{\sharp}{\rightarrow} Y^{\prime} \rightarrow Y^{\prime \prime} \rightarrow 0$ is an Auslander-Reiten sequence in $\bmod _{\mathrm{sp}}(\hat{S})$ and $Y, Y^{\prime \prime}$ are indecomposable. In order to prove that $L_{\eta} \mathfrak{V}$ is an Auslander-Reiten sequence it is sufficient to show that $L_{\hat{\eta}}(u)$ is a left almost split map. Let $g: L_{\hat{\eta}} Y \rightarrow X$ be a nonzero nonisomorphism in $\operatorname{ind}_{\mathrm{sp}}(R)$. It follows from (a) that $X \cong L_{\hat{\eta}} U$ for some $U$ in ind $\mathrm{d}_{\mathrm{pp}}(\hat{S})$ and we get a factorization of $g$ through $\boldsymbol{L}_{\hat{\eta}}(u)$ because $\boldsymbol{L}_{\hat{\eta}}$ is fully faithful and $u$ is left almost split. The proof for the functor $\boldsymbol{T}_{\xi}$ is similar.

Now suppose that $\mathfrak{X} ; 0 \rightarrow X \rightarrow X^{\prime} \rightarrow X^{\prime \prime} \rightarrow 0$ is an Auslander-Reiten sequence in $\bmod _{\mathrm{sp}}(R)$ and $X$ is non-sp-injective indecomposable. If $X \cong L_{\eta} Y$ then by Lemma 3.9(b), $Y$ is non-sp-injective indecomposable and therefore there exists an Auslander-Reiten sequence $\mathfrak{Y})$ in $\bmod _{\mathrm{Bp}}(\hat{S})$ starting with $Y$. Since we know that $L_{\eta} \mathfrak{Y}$ is an Auslander-Reiten sequence we have $\mathfrak{X} \cong L_{A}(2)$.

Next suppose that $X \notin \operatorname{Im} L_{\eta}$. It follows from (a) that $X \cong T_{\xi} Z$ for some $Z$ and $X$ is not isomorphic to a module of the form $\boldsymbol{T}_{\xi} H_{j-1}^{i}$. Hence in view of Lemma 3.9(b), $Z$ is not sp-injective and therefore there exists an Auslander-Reiten sequence 3 in $\bmod _{\mathrm{sp}}(T)$ starting with $Z$. Since we know that $T_{\xi}, 3$ is an Auslander-Reiten sequence, $\mathfrak{X} \cong \boldsymbol{T}_{\xi}, 3$ and the proof is complete.

Following Ringel [17] we call $\operatorname{Irr}\left(X, X^{\prime}\right)=\operatorname{Hom}_{R}\left(X, X^{\prime}\right) / \operatorname{rad}^{2}\left(X, X^{\prime}\right)$ the $E\left(X^{\prime}\right)-E(X)$-bimodule of irreducible maps from $X$ to $X^{\prime}$, where $E(X)=\operatorname{End}(X) / J \operatorname{End}(X)$. 
As a consequence of the results above we get

COROLlaRY 3.11. Let $R$ be as in Theorem 3.10. Then

(a) $R$ is not sp-sincere if one of $\mathscr{2}^{\prime}, \mathscr{Q}^{\prime \prime}$ is not empty.

(b) The natural bimodule epimorphisms $\tilde{\boldsymbol{L}}_{\hat{\eta}}: \operatorname{Irr}\left(Y, Y^{\prime}\right) \rightarrow \operatorname{Irr}\left(L_{\hat{\eta}} Y, L_{\hat{\eta}} Y^{\prime}\right), \widetilde{\boldsymbol{T}}_{\xi}: \operatorname{Irr}\left(Z, Z^{\prime}\right)$ $\rightarrow \operatorname{Irr}\left(\boldsymbol{T}_{\xi} Z, T_{\xi} Z^{\prime}\right)$ are isomorphisms for $Y, Y^{\prime}$ in $\operatorname{ind}_{\mathrm{sp}}(\hat{S})$ and $Z, Z^{\prime}$ in $\operatorname{ind}_{\mathrm{sp}}(T)$.

(c) $\Gamma_{\mathrm{sp}}(R)=L_{\hat{\eta}} \Gamma_{\mathrm{sp}}(\hat{S}) \cup \boldsymbol{T}_{\xi} \Gamma_{\mathrm{sp}}(T)$ is obtained from $\Gamma_{\mathrm{sp}}(\hat{S})$ and $\Gamma_{\mathrm{sp}}(T)$ by the identification of the final hereditary sp-injective section $H_{i_{i}}^{i} \rightarrow \ldots \rightarrow H_{0}^{i}$ in $\Gamma_{\mathrm{sp}}(T)$ with the starting hereditary projective section $P_{t_{i}+1}^{i} \rightarrow \ldots \rightarrow P_{1}^{i}$ in $\Gamma_{\mathrm{sp}}(\hat{S})$ for $i=1, \ldots, u$ (see 3.8 and Fig. 7 comp. [27]).

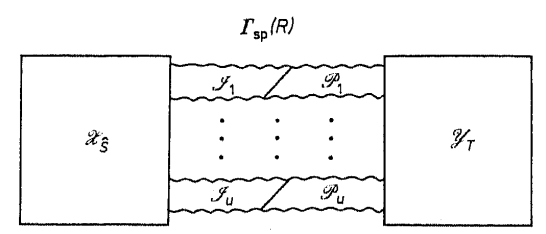

Fig. 7

Applying the same type of argument as in the proof of Theorem 3.10(a) we get

Proposition 3.12. Suppose that $R=F(\mathscr{Q}, \Omega)$ and $(\mathscr{2}, \Omega)$ admits a triangular decomposition (3.2) and a set $\mathscr{P} \subseteq \mathbf{p}\left(\mathscr{2}^{\prime \prime}\right)$ satisfying 3.3(a) and the following condition:

(S) If $i \in \mathscr{Q}_{0}^{\prime}$ and $Z$ is in $\operatorname{ind}_{\mathrm{sp}}(T)$ (see (3.4)) such that $\operatorname{Hom}_{T}\left(\boldsymbol{r}_{\xi}\left(e_{i} R\right), Z\right) \neq 0$ then $\left.Z\right|_{\mathscr{2}^{\prime \prime}}=Z f$ is a direct sum of copies of $E_{A}\left(e_{p} A\right), p \in \mathscr{P}$ (see 3.7).

Then every $X$ in $\operatorname{ind}_{\mathrm{sp}}(R)$ belongs either to $\operatorname{Im} L_{\hat{\eta}}$ or to $\operatorname{Im} T_{\xi}$.

Let us finish this section by a generalization of the Ringel-Roggenkamp [19] edge reduction.

COROLLARY 3.13. Let $R=F(\mathscr{Q}, \Omega)$ be a right multipeak algebra such that $(\mathscr{2}, \Omega)$ is a disjoint union of $\left(\mathcal{Q}^{\prime}, \Omega^{\prime}\right)$ and a quiver

$$
\mathscr{B}: p \leftarrow \ldots \leftarrow 0 \rightarrow \ldots \rightarrow 0 \leftarrow \ldots
$$

of type $A_{n}$ having $\geqslant 1$ sinks. Suppose that there is no path $c \rightarrow i \in \mathscr{Q}_{0}^{\prime}, c \in \epsilon^{\prime} \mathscr{C}_{0}^{\prime}$, and there is an edge $\gamma: s \rightarrow p$, $s$ a sink in $\mathscr{Q}^{\prime}$, such that any nonzero path $i \rightarrow c \in \mathscr{C}_{0}, i \in \mathscr{Q}_{0}^{\prime}$, in $(\mathscr{Q}, \Omega)$ has a factorization through $\gamma$. Then any $X$ in $\operatorname{ind}_{\mathrm{sp}}(R)$ is in the image of one of the functors

$$
\bmod _{\mathrm{sp}} F(\mathcal{L}-\{s\}, \Omega) \stackrel{\boldsymbol{L}^{s}}{\longrightarrow} \bmod _{\mathrm{sp}}(R) \stackrel{{ }^{T^{s}}}{{ }^{*}} \bmod _{\mathrm{sp}} F \mathscr{C}
$$

where $\boldsymbol{T}^{s}=\boldsymbol{T}_{g}, g=\sum_{\mathrm{\epsilon} \in \mathbb{8}} e_{c}$, is the natural embedding and $\boldsymbol{L}^{s}=\boldsymbol{L}_{e}, e=\sum_{i \neq s} e_{i}$.

Proof. Similarly to the proof of Theorem 3.10 one can show that if $X$ is in $\operatorname{ind}_{\mathrm{sp}}(R)$ and $X(\gamma): X e_{s} \rightarrow X e_{p}$ is nonzero then $X(\gamma)$ is bijective and according to Lemma 3.7, $X \in \operatorname{Im} \boldsymbol{L}^{s}$. Hence the corollary follows.
We shall show that the splitting theorem remains true for a class of artinian rings under a suitable modification of Definition 3.3

For this purpose we suppose that $R=\bigoplus_{i \in I_{R}} e_{i} R$ is a basic artinian right multipeak ring, we keep the notation of Section 2 and given $i \neq j$ we set

$$
d_{i j}=\text { length }\left(e_{i} R e_{j}\right) e_{j} R e_{j}, \quad d_{i j}^{\prime}=\text { length }_{e_{i} R e_{i}}\left(e_{i} R e_{j}\right) .
$$

Defintrion 3.14. The ring $R$ has a splitting decomposition if there exist a set $\mathscr{P}=\left\{p_{1}, \ldots, p_{u}\right\} \cong p\left(I_{R}\right)$ and a disjoint union decomposition

$$
I_{R}=I^{\prime} \dot{\cup} C \dot{\cup} I^{\prime \prime}
$$

such that $C=C^{1} \dot{\cup} \ldots \dot{\cup} C^{\prime \prime} \subseteq I_{R}-p\left(I_{R}\right), \mathscr{P} \subseteq I^{\prime \prime}, I^{\prime \prime}$ is not empty and the following conditions are satisfied:

(a) $\hat{S}=\hat{\eta} R \hat{\eta}$ and $T=\xi R \xi$ are right multipeak rings with peak idempotents $e_{p}$, $p \in p\left(I_{R}\right) \cap I^{\prime} \cup \mathscr{P}$, and $e_{q}, q \in p(I) \cap I^{\prime \prime}$, respectively, where $\hat{\eta}=e_{p_{1}}+\ldots+e_{p_{u}}+\sum_{i \in I^{\prime} \cup C} e_{i}$ and $\xi=\sum_{j \in I^{\prime \prime}} e_{j}$.

(b) $d_{i j} d_{i j}^{\prime}=1$ for $i, j \in \hat{C}^{t}:=C^{t} \cup\left\{p_{t}\right\}, t=1, \ldots, u$.

(c) $d_{i j}=0$ if either $i \in C^{t}, j \in C^{s}, t \neq s$, or $i \in C \cup I^{\prime \prime}, j \in I^{\prime}$, or $i \in I^{\prime \prime}, j \in C$, or $i \in C$, $j \in \boldsymbol{p}\left(I_{R}\right)-\mathscr{P}$, or $i \in I^{\prime}, j=\left(p\left(I_{R}\right) \cap I^{\prime \prime}\right)-\mathscr{P}$.

(d) $d_{i j}=\sum_{p \in i f i p} d_{i p} d_{j p}^{\prime}$ for any $i \in I^{\prime}$ and $j \in I^{\prime \prime}$.

In this case we have the induced functors (3.6) and by a modification of the arguments in the proof of our splitting results above and those in $[11,12]$ we easily prove

THeOrem 3.15. Let $R$ be a basic artinian right multipeak PI-ring (2.0) such that $\bmod _{\mathrm{sp}}(R)$ has Auslander-Reiten sequences. If $R$ admits a splitting decomposition induced by $\mathscr{P}$ and $I_{R}=I^{\prime} \cup C \cup I^{\prime \prime}$ then in the notation above the statements (a) and (b) in Theorem 3.10 as well as (a)--(c) in Corollary 3.11 are true.

\section{Examples and concluding remarks.}

4.0. Multipeak posets. Suppose that $I$ is a finite poset and consider $I$ as a bound quiver whose arrows are pairs $(i, j)$, where $i \prec j$ and there is no $t \neq i, j$ such that $i \prec t \prec j$. The relations are the natural commutativity ones. Suppose that

$$
I=I^{\prime}+\left(C^{1}+\ldots+C^{\prime \prime}\right)+\left(p_{1}^{\nabla}+\ldots+p_{\| \prime}^{\nabla}\right)
$$

is a disjoint union triangular poset decomposition, where $p_{1} \ldots, p_{u} \in \boldsymbol{p}(I)$ and $C^{1}, \ldots, C^{u}$ are linearly ordered subposets of $I-p(I)$ (see (3.2)). The decomposition is splitting in the sense of 3.3 if and only if all clements $u \in C^{i}+p_{i}^{\nabla}, v \in C^{j}+p \gamma$ are unrelated for $i \neq j$, and $s \prec p_{i}, s \in I^{\prime}$, implies $s \prec r$ for all $r \prec p_{i}$, where $i=1, \ldots, u$.

If $I$ is a one-peak poset, then $u=1, p_{1}$ is the unique maximal element in $I$ and the definition above is the usual one given by Nazarova and Roiter in [15]. For $I$ arbitrary our definition is a natural extension of that given in [15]. The situation is more complicated for multipeak posets with zero relations. As a simple example consider the two-peak poset $I$ of Fig. 8 with zero relations $\alpha \beta=\gamma \delta=\varepsilon \xi=0$. The 
decomposition

$$
I=I^{\prime}+C^{1}+C^{2}+I^{\prime \prime}
$$

where $I^{\prime}=\{a, b \rightarrow d\}, C^{1}=\left\{c_{1} \rightarrow c_{2}\right\}, C^{2}=\left\{c_{3} \rightarrow c_{4}\right\}, I^{\prime \prime}=I-\left(C^{1}+C^{2}+I^{\prime}\right)$, is obviously splitting.

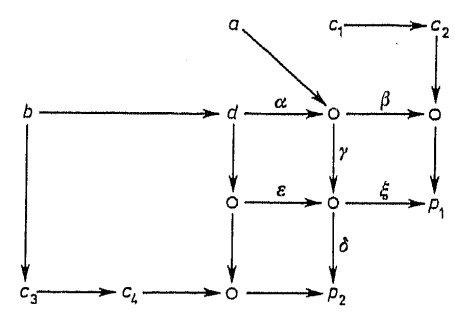

Fig. 8

4.1. The following two examples are not of the poset type. They are representative for the Galois covering reduction of nonschurian right peak algebras mentioned in the Introduction.

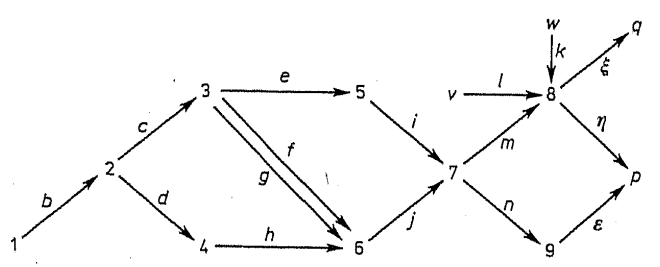

Fig. 9

(a) Consider a right two-peak algebra $R=F(\mathscr{Q}, \Omega)$, where 2 is shown in Fig. 9 and $\Omega=\left(c g-d h, e i-f j, m \eta-n \varepsilon, h j m \xi\right.$, gjm $\xi$, in, $k \xi$, l $\eta$. If we set $\mathscr{Q}_{0}^{\prime}=\{1,2,3,4\}, \mathscr{C}_{0}^{1}=\{u\}$, $\mathscr{C}_{0}^{2}=\{v\}, \mathscr{Q}_{0}^{\prime \prime}=\{5,6,7,8,9, p, q\}$ then

$$
\mathscr{Q}_{0}=\mathscr{2}_{0}^{\prime}+\mathscr{C}_{0}^{1}+\mathscr{C}_{0}^{2}+\mathscr{Q}_{0}^{\prime \prime}
$$

defines a splitting decomposition of $(2, \Omega)$ with $u=2, p_{1}=p$ and $p_{2}=q$. Note that the algebras (3.4)

$$
\hat{S}=F\left(\mathscr{Q}_{0}^{\prime}+\hat{\mathscr{C}}^{1}+\hat{\mathscr{C}}^{2}, \Omega \mid\right), \quad T=F\left(\mathscr{C}^{1}+\mathscr{C}^{2}+\mathscr{Q}^{\prime \prime}, \Omega \mid\right)
$$

are the bound quiver algebras of the quivers of Fig. 10 with all commutativity relations and the following zero relations: $5 \rightarrow-\rightarrow, v \rightarrow-p, w \rightarrow q$ in the right hand quiver. It follows that $\hat{S}$ and $T$ have the separation property for radicals of indecomposable projective


Fig. 10

right modules and $\Gamma_{\mathrm{sp}}(S), \Gamma_{\mathrm{sp}}(T)$ have a preprojective and a preinjective component (see [26; Proposition 4.7]). Modifying the arguments used in $[12,24]$ one can show that



Fig. 11

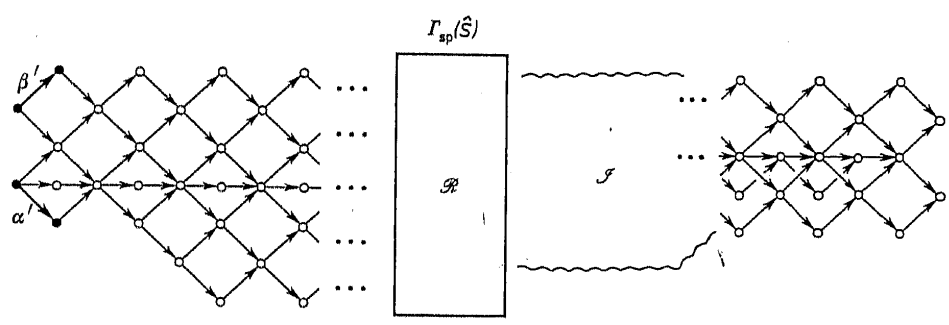

Fig. 12

$\Gamma_{\mathrm{sp}}(T)$ and $\Gamma_{\mathrm{sp}}(\hat{S})$ have the forms presented in Figures 11 and 12, respectively. By [25; Theorem 3.4 and Corollary 3.13] there is an equivalence of categories

$$
\left.\bmod _{\mathrm{sp}}(S) / \mathscr{L} \cong J \text {-sp/[e } e_{1} F J^{*}, e_{2} F J^{*}, e_{3} F J^{*}\right]
$$

where $\mathscr{L}$ is the ideal in $\bmod _{s p}(\hat{S})$ generated by $e_{j} \hat{S}, j=v, q, p, 4, w$ and by the submodule $e_{4} \hat{S}+e_{w} \hat{S}$ of $E\left(e_{p} \hat{S}\right)$, and $f^{*}$ is the poset of Fig. 13. Hence if $F=\bar{F}$, the category $\bmod _{\mathrm{sp}}(\hat{S})$

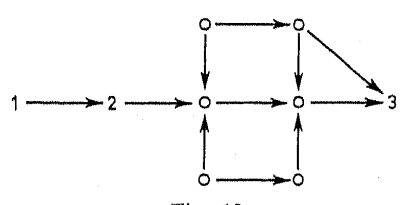

Fig. 13 
is of tame type [6] because $J^{*}$ is of tame type by the criterion of Nazarova [14]. It follows from Corollary 3.11 that

$$
\Gamma_{\mathrm{sp}}(R)=\Gamma_{\mathrm{sp}}(T) \cup \Gamma_{\mathrm{sp}}(\hat{S}) /\left(\alpha \equiv \alpha^{\prime}, \beta \equiv \beta^{\prime}\right) .
$$

The part $\mathscr{R}$ in Figure 12 consists of tubes [17]. The category $\bmod _{\mathrm{sp}}(R)$ is of tame type. Note also that the algebras $\hat{S}$ and $T$ are simply connected [0], whereas $R$ is not
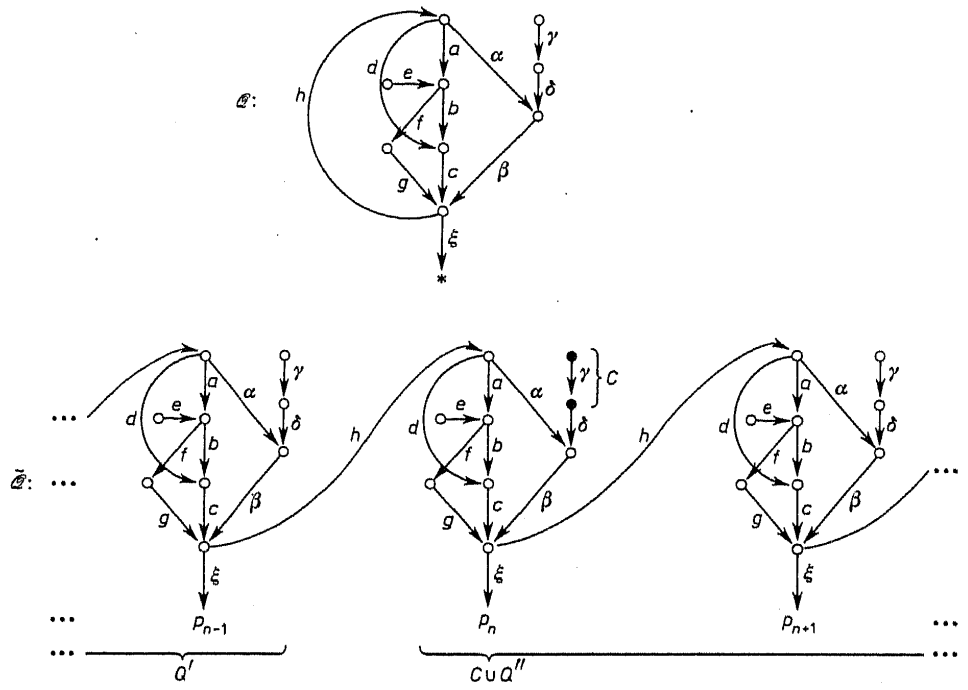

Fig. 14
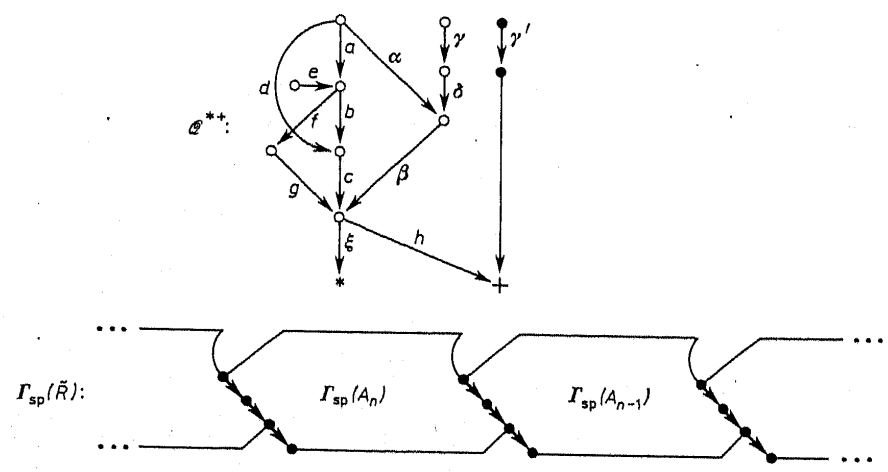

Fig. 15 (b) Consider the right peak algebra $R=F(2, \Omega)$ and the right multipeak algebra $\tilde{R}=F(\tilde{\mathscr{Q}}, \tilde{\Omega})$, where $\mathscr{Q}$ and $\tilde{\mathscr{Q}}$ are the quivers in Fig. 13 and

$$
\Omega=\tilde{\Omega}=(d c-a f g, a b c-d c-\alpha \beta, a b c \xi-\alpha \beta \xi,
$$

$e f g-e b c, h \alpha \beta-h a b c, b c h-f g h, \beta h, h d, h a f, g \xi)$.

Note that $(\tilde{\mathscr{Q}}, \tilde{\Omega})$ is the universal cover of $(\mathscr{L}, \Omega)$ and $\mathscr{Q}^{\prime}, \mathscr{C} \cup \mathscr{Q}^{\prime \prime}, \mathscr{C}=\{\bullet \rightarrow \bullet\}$ marked in Fig. 14 induce a splitting decomposition of $(\widetilde{\mathscr{Q}}, \tilde{\Omega})$ for any fixed $n$. By repeating the splitting procedure we easily conclude from Theorem 3.10 that the coordinate support of any $X$ in $\operatorname{ind}_{s p}(\widetilde{R})$ is a subquiver of the quiver $\left(2^{*+}, \widetilde{\Omega} \mid\right)$ in Fig. 15. The two-peak algebra $A=F\left(2^{*+}, \tilde{\Omega}\right)$ is sp-representation-infinite according to the criterion of Weichert [29]. It follows that $\tilde{R}$ is locally sp-representation-infinite and by [22; Theorem 1.10] $R$ is sp-representation-infinite. Moreover, we conclude from Corollary 3.11 that $\Gamma_{\mathrm{sp}}(\tilde{R})$ is obtained from $\Gamma_{\mathrm{sp}}\left(A_{n}\right), A_{n}=A, n=0, \pm 1, \pm 2, \ldots$ by successive glueing presented in Fig. 15, whereas $\Gamma_{\mathrm{sp}}(R)$ is obtained from $\Gamma_{\mathrm{sp}}(A)$ by simple glueing of two linear three-vertex sections like in $[24 ; 5.15]$. Since $A$ obviously has the separation property [26], $\Gamma_{\mathrm{sp}}(A)$ has a preprojective component $\mathscr{P}_{\mathrm{sp}}(A)$ which can be easily computed by the well-known preprojective component construction like in $[12,24]$.

4.2. Multiserial multipeak trees. A multipeak bound quiver $(2, \Omega)$ will be called a multiserial tree if 2 is a tree, $\Omega$ consists of zero relations and the following conditions are satisfied (comp. [7; 5.2] and [5]):

(i) For every edge $a$ in $\mathscr{2}_{1}$ there exists at most one edge $b$ and at most one edge $c$ such that $a c, b a$ are not in $\Omega$.

(ii) For any $p \in p(\mathscr{Q})$ there are chains $\mathscr{C}_{p}^{\prime}, \mathscr{C}_{p}^{\prime \prime}$ such that $p^{\nabla}=\mathscr{C}_{p}^{\prime} \cup \mathscr{C}_{p}^{\prime \prime}, p \in \mathscr{C}_{p}^{\prime}$, $\mathscr{C}_{p}^{\prime} \cap \mathscr{C}_{p}^{\prime \prime}=\varnothing$ and $\mathscr{C}_{p}^{\prime \prime} \cap q^{\nabla}$ is empty if $p \neq q \in \boldsymbol{p}(\mathscr{2})$. We put $\hat{\mathscr{C}}_{p}^{\prime \prime}=\mathscr{C}_{p}^{\prime \prime} \cup\{p\}$.

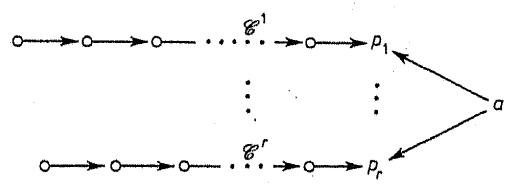

Fig. 16

If $\mathscr{C}^{1}, \ldots, \mathscr{C}^{r}$ are pairwise disjoint chains and $\left|\mathscr{C}_{0}^{1}\right|=m_{1}, \ldots,\left|\mathscr{C}_{0}\right|=m_{\mathrm{r}}$ then we denote by $T_{a}\left(\mathscr{C}^{1}, \ldots, \mathscr{C}^{r}\right)=T\left(m_{1}, \ldots, m_{r}\right)$ the tree of Fig. 16. We say that $T\left(m_{1}, \ldots, m_{r}\right)$ is a peak subtree of $(2, \Omega)$ if there is a bound quiver embedding of $T\left(m_{1}, \ldots, m_{r}\right)$ in $(\mathscr{Q}, \Omega)$ which carries peak vertices to peak ones and oriented edges to nonzero oriented paths in $(2, \Omega)$.

Propositron. Let $R=F(\mathscr{Q}, \Omega)$, where $F$ is a division ring and $(2, \Omega)$ is a locally finite multiserial multipeak tree.

(a) The radical of any indecomposable projective right $R$-module is a direct sum of uniserial modules. If $X$ is in ind $_{s p}(R)$ then $\operatorname{csup}(X)=T_{a}\left(\mathscr{C}_{p_{1}}, \ldots, \mathscr{C}_{p_{r}}\right)$ for some $a \in \mathscr{Q}_{0}$, $p_{1}, \ldots, p_{r} \in p(\mathscr{2})$ and $\mathscr{C}_{p_{1}} \subseteq \mathscr{\mathscr { C }}_{p_{1}}^{\prime \prime}, \ldots, \mathscr{C}_{p_{r}} \subseteq \hat{\mathscr{C}}_{p_{r}}^{\prime \prime}$ with $p_{i} \in \mathscr{C}_{p_{i}}$ 
(b) $R$ is locally sp-representation-finite if and only if $(2, \Omega)$ does not contain peak subtrees of the form $\boldsymbol{T}(1,1,1,1), \boldsymbol{T}(2,2,2), \boldsymbol{T}(1,3,3), \boldsymbol{T}(1,2,5)$.

(c) $R$ is locally sp-representation-tame if and only if $(2, \Omega)$ does not contain peak subtrees of the form $\boldsymbol{T}(1,1,1,1,1), \boldsymbol{T}(1,1,1,2), \boldsymbol{T}(2,2,3), \boldsymbol{T}(1,3,4)$ and $\boldsymbol{T}(1,2,6)$.

Proof. (a) The first part follows immediately from the definition. In order to prove the second part one can suppose without loss of generality that $(2, \Omega)$ is finite sp-sincere and $\operatorname{csup}(X)=(\mathscr{Q}, \Omega)$. Let $p(2)=\left\{p_{1}, \ldots, p_{r}\right\}$ and let $a$ be a minimal vertex in $\mathscr{C}_{p 1}^{\prime} \cup \ldots \cup \mathscr{C}_{p r}^{\prime}$ (see (ii)). Suppose that $p_{1}, \ldots, p_{s}, s \leqslant r$, are all $p \in p(\mathscr{2})$ such that there is a nonzero path $\gamma: a \rightarrow p$ in $(2, \Omega)$. We shall prove (a) by showing that $s=r$, and $T_{a}\left(\hat{\mathscr{C}}_{p_{1}}^{\prime \prime}, \ldots, \hat{\mathscr{C}}_{p r}^{\prime \prime}\right)=(2, \Omega)$. For this purpose consider the decomposition 3.3 with $u=s$, $\mathscr{Q}_{0}^{\prime}=\{a\}, \mathscr{C}^{j}=\mathscr{C}_{p_{j}}^{\prime \prime}, j=1, \ldots, s$, and $\mathscr{Q}^{\prime \prime}=\mathscr{Q}-\left(p_{1}^{\nabla} \cup \ldots \cup p_{s}^{\nabla}\right)$. Since $a$ is minimal and $(2, \Omega)$ is a multiserial tree, the above decomposition is splitting, and since $R$ is sp-sincere, by Corollary 3.11(a) the set $\mathscr{Q}_{0}^{\prime \prime}$ is empty. Hence $s=r,(\mathscr{Q}, \Omega)$ $=T_{a}\left(\hat{\mathscr{C}}_{p_{1}}^{\prime \prime}, \ldots, \hat{\mathscr{C}}_{p_{r}}^{\prime \prime}\right)$ and (a) follows.

It follows from (a) that $R$ is locally sp-representation-finite (resp. -tame) if and only if for every peak subtree $T_{a}\left(\hat{\mathscr{C}}_{p_{1}}^{\prime \prime}, \ldots, \hat{\mathscr{C}}_{p_{r}}^{\prime \prime}\right)$ of $(\mathscr{Q}, \Omega)$ the right multipeak hereditary algebra $R_{a}\left(m_{1}, \ldots, m_{r}\right)=F \boldsymbol{T}_{a}\left(\hat{\mathscr{C}}_{p_{1}}^{\prime \prime}, \ldots, \hat{\mathscr{C}}_{p_{r}}^{\prime \prime}\right)$ is sp-representation-finite (resp. -tame), where $m_{j}$ is the number of yertices in ' $\hat{\mathscr{C}}_{p_{j}}^{\prime \prime}$. Consider the poset $I\left(m_{1}, \ldots, m_{r}\right)=T_{a}\left(\hat{\mathscr{C}}_{p_{1}}^{\prime \prime}, \ldots, \hat{\mathscr{C}}_{p_{r}}^{\prime \prime}\right)-\{a\}$. It follows from [25; Corollary 3.13] applied to $I=T_{a}\left(m_{1}, \ldots, m_{r}\right), c=a$, that $\xi_{c} I=I\left(m_{1}, \ldots, m_{r}\right)$ and there is a full dense functor

$$
\text { ad: } \bmod _{\mathrm{sp}}\left(R_{a}\left(m_{1}, \ldots, m_{r}\right)\right) \rightarrow\left(I\left(m_{1}, \ldots, m_{r}\right)-\mathrm{sp}\right) /\left[P_{*}\right]
$$

preserving the representation type and such that $\operatorname{Ker} a d=\left[I\left(m_{1}\right)\right.$-sp, $\ldots, I\left(m_{r}\right)$-sp $]$, where $I$-sp means the category of $I$-spaces, $P_{*}$ is the unique simple projective $I$-space and $I\left(m_{j}\right)$-sp is considered as a full subcategory of $\bmod _{\mathrm{sp}}\left(R_{a}\left(m_{1}, \ldots, m_{\mathrm{r}}\right)\right)$ in a natural way. Consequently, the problem reduces to the problem for $I\left(m_{1}, \ldots, m_{r}\right)$-spaces and therefore (b) is a consequence of the criterion of Kleiner [10], whereas (c) is a consequence of the criterion of Nazarova [14]. The proof is complete.

For an illustration of the result above let us consider the right peak algebra

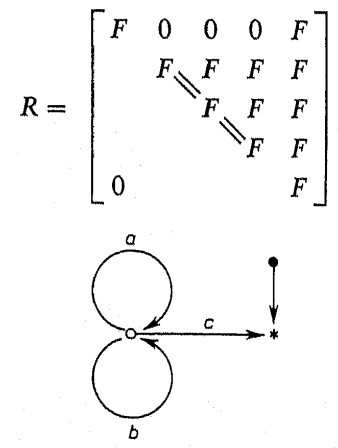

Fig. 17 where $F$ is an algebraically closed field. Note that $R=F(\mathscr{Q}, \Omega)$ where $\mathscr{2}$ is shown in Fig. 17 and $\Omega: a^{2}=b^{2}=b a=a c=0$. The infinite tree $\mathscr{Q}$ in Fig. 18 with relations $\tilde{\Omega}=\Omega$ is a Galois cover of $(\mathscr{Q}, \Omega)$ with the free group $G=Z * Z$. Since $(\tilde{\mathscr{Q}}, \tilde{\Omega})$ is a multiserial

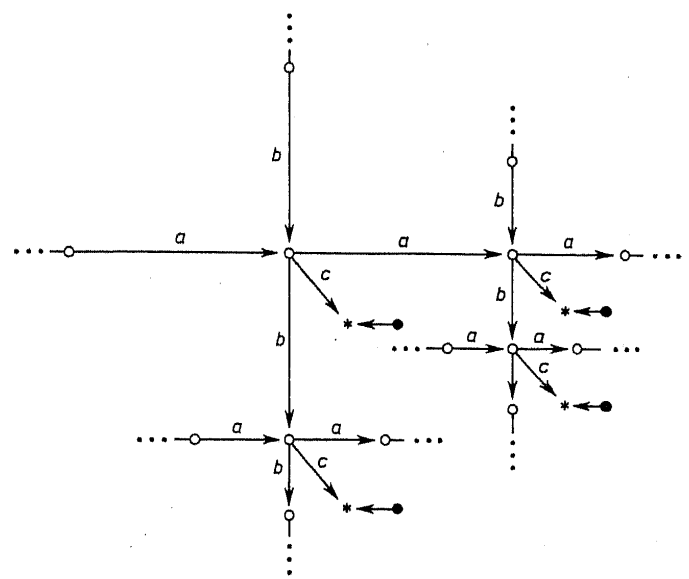

Fig. 18

multipeak tree we conclude from Proposition 4.2 that the coordinate support of every indecomposable in $\bmod _{\mathrm{sp}} F(\tilde{\mathcal{Q}}, \tilde{\Omega})$ is a subquiver of $\boldsymbol{A}=\boldsymbol{T}(2,2,2)$ (see Fig. 19) and $F(\widetilde{\mathscr{Q}}, \tilde{\Omega})$ is locally sp-representation-tame [6]. Hence by [22; Theorem 1.10], [24; Theorem 0.2$]$ and the arguments applied in $[6,7]$ the category $\bmod _{\mathrm{sp}}(R)$ is of tame type.

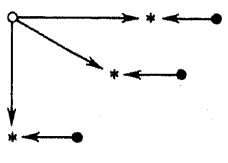

Fig. 19

Since $F \Delta$ is a hereditary algebra of Euclidean type $\widetilde{E}_{6}$ it is tame and $\bmod (F \Delta)$ as well as $\Gamma(F \Delta)$ are well known. Since every $X$ in $\operatorname{ind}(F \Delta)-\operatorname{ind}_{\mathrm{sp}}(F \Delta)$ is simple injective the category $\bmod _{\mathrm{sp}}(F \Delta)$ can be completely described and the restriction of the push-down functor to $\bmod _{\mathrm{sp}}(F \Delta)$ yields a description of $\bmod _{\mathrm{sp}}(R)$. By the arguments used in $[26$; Section 5] one can show that $\bmod _{\mathrm{sp}}(R)$ is representation equivalent to a cofinite subcategory of $\bmod _{\mathrm{sp}}(F \Delta)$.

Let us finish by an example which illustrates the generalized splitting theorems given in Proposition 3.12 and Theorem 3.15.

EXAmple 4.3. For $t>0$ consider the two-peak algebra $R_{t}=F(\mathscr{Q}, \Omega)$, where $F$ is 
a division ring, $\mathscr{Q}$ is the quiver

$$
\begin{aligned}
& 0 \rightarrow 0 \rightarrow \ldots \rightarrow 0 \rightarrow 0 \rightarrow p \\
& \uparrow a_{t+1} \\
& 1 \frac{a_{2}}{b_{2}} 2 \frac{a_{3}}{\overrightarrow{b 3}} 3 \rightrightarrows \ldots \rightrightarrows t-1 \frac{a_{t}}{\overrightarrow{b_{t}}} t \\
& 0 \rightarrow 0 \rightarrow \ldots \rightarrow 0 \rightarrow 0 \rightarrow q
\end{aligned}
$$

and $\Omega=\left(a_{2} b_{3}, b_{2} a_{3}, a_{3} b_{4}, b_{3} a_{4}, \ldots, a_{t} b_{t+1}, b_{t} a_{t+1}\right)$. Let $\mathscr{Q}^{\prime}$ and $2^{\prime \prime}$ be the full subquivers consisting of points $1, \ldots, t-1$ and $t, p, q$ respectively, and let $\mathscr{C}^{p}$ and $\mathscr{C}^{\prime \prime}$ be the top chain and the bottom chain in $\mathscr{Q}$, respectively. Then $\mathscr{Q}=\mathscr{Q}^{\prime} \cup \mathscr{C}^{p} \cup \mathscr{C}^{q} \cup \mathscr{Q}^{\prime \prime}$ satisfies the assumptions in Proposition 3.12 and in Theorem 3.15 with $u=2, p_{1}=p, p_{2}=q$, there are $F$-algebra isomorphisms $\hat{S} \cong \hat{\eta} R_{t} \hat{\eta} \cong R_{t-1}, T \cong \xi R_{t} \xi \cong R_{1}$ and it follows by induction that $\bmod _{\mathrm{sp}}\left(R_{t}\right)$ is of finite type for all $t \geqslant 1$.

\section{References}

[0] I. Assem and A. Skowroński, On some classes of simply connected algebras, Proc. London Math. Soc. 56 (1988), 417-450.

[1] M. A uslander, Representation theory of artin algebras, I, Comm. Algebra 1 (1974), 177-268.

[2] M. Auslander and S. O. Smalø, Almost split sequences in subcategories, J. Algebra 69 (1981), 426-454.

[3] R. B autista and D. Simson, Torsionless modules over l-hereditary 1-Gorenstein artinian rings, Comm. Algebra 12 (1984), 899-936.

[4] K. Bongartz and P. Gabriel, Covering spaces in representation theory, Invent. Math. 65 (1982), 331-378.

[5] M. Dembiński, P. Dowbor and A. Skowroniski, On indecomposable representations of quivers with zero-relations, Fund. Math. 130 (1988), 169-180.

[6] P. Dowbor and A. Skowronski, On the representation type of locally bounded categories, Tsukuba J. Math. 10 (1986), 63-72.

[7],,-- , Galois coverings of representation-infinite algebras, Comment. Math. Helv. 62 (1987),

[8] E. L. Green, Group-graded algebras and the zero relation problem, in: Lecture Notes in Math. 903, Springer, 1981, 106-115.

[9] D. Hughes and J. W a sch büsch, Trivial extensions of tilted algebras, Proc. London Math. Soc. 46 (1983), 347-364.

[10] M. M. Kleiner, Partially ordered sets of finite type, Zap. Nauchn. Sem. LOMI 28 (1972), 32-41 (in Russian).

[11] B. Klemp and D. Simson, Schurian sp-representation-finite right peak PI-rings and their indecomposable socle projective modules, J. Algebra 134 (1990), 390--468.

[12] -, -, On the Auslander-Reiten valued quiver of right peak rings, Fund. Math. 136 (1990), 91-114.

[13] R. Martínez and J. A. de la Peña, The universal cover of a quiver with relations, J. Pure Appl. Algebra 30 (1983), 277-292

[14] L. A. Naza rova, Partially ordered sets of infinite type, Izv. Akad. Nauk SSSR Ser. Mat. 39 (1975), 963-991 (in Russian).

[15] L. A. Nazarova and A. V. Roiter, Representations of partially ordered sets, Zap. Nauchn.
[16],,-- , Representations of bipartite completed posets, Comment. Math. Helv. 63 (1988),

17] C. M. Ringel, Tame Algebras and Integral Quadratic Forms, Lecture Notes in Math. 1099, Springer, 1984

[18] - , Hall polynomials for the representation-finite hereditary algebras, Adv. in Math. 84 (1990), $137-178$.

[19] C. M. Ringel and K. W. Roggenkamp, Socle-determined categories of representations of hereditary tensor algebras, J. Algebra 64 (1980), 249-269.

[20] K. W. Roggenk amp, Indecomposable representations of orders, in: Topics in Algebra, Part 1: Rings and Representations of Algebras, Banach Center Publ. 26, PWN, Warszawa 1990, 449-491.

[21] D. Simson, Vector space categories, right peak rings and their socle projective modules, J. Algebra 92 (1985), 532-571.

[22] -, Socle reductions and socle projective modules, ibid. 103 (1986), 18-68.

[23] -, Moduled categories and adjusted modules over traced rings, Dissertationes Math. 269 (1990).

[24] -, Representations of bounded stratified posets, coverings and socle projective modules, in: Topics in Algebra, Part 1: Rings and Representations of Algebras, Banach Center Publ. 26, PWN, Warszawa 1990, 499-533.

[25] -, Peak reductions and waist reflection functors, Fund. Math. 137 (1991), 115-145.

[26] -, Right peak algebras of two-separate stratified posets, their Galois coverings and socle projective modules, to appear.

[27] -, On the representation type of stratified posets, C. R. Acad. Sci. Paris 311 (1990), 5-10.

[28] D. Sims on and A. Skow roński, Extensions of artinian rings by hereditary injective modules, in: Lecture Notes in Math. 903, Springer, 1981, 315-330.

[29] Th. Weichert, Darstellungstheorie von Algebren mit projektivem Sockel, Doctoral Thesis, Universität Stuttgart, 1989.

INSTITUTE OF MATHEMATICS

NICHOLAS COPERNICUS UNIVERSITY

Chopina $12 / 18$

Poland

Received 10 January 1990; in revised form 10 May 1990 Sem. LOMI 28 (1972), 5-31 (in Russian). 